\title{
Conversion of relativistic pair energy into radiation in the jets of active galactic nuclei
}

\author{
R. Schlickeiser ${ }^{1}$, R. Vainio ${ }^{2}$, M. Böttcher ${ }^{3,4,6}$, I. Lerche ${ }^{5}$, M. Pohl ${ }^{1}$, and C. Schuster ${ }^{1}$ \\ 1 Institut für Theoretische Physik, Lehrstuhl IV: Weltraum- und Astrophysik, Ruhr-Universität Bochum, \\ 44780 Bochum, Germany \\ 2 Space Research Laboratory, Department of Physics, Turku University, 20014 Turku, Finland \\ 3 Department of Physics and Astronomy, Rice University, MS 108, 6100 S. Main Street, Houston, \\ TX 77005 - 1892, USA \\ ${ }^{3}$ Department of Physics and Astronomy, Ohio University, Clippinger 339, Athens, OH 45701, \\ USA (current address) \\ ${ }^{4}$ Department of Geological Sciences, University of South Carolina, Columbia, SC 29208, USA \\ 5 Chandra Fellow
}

Received 30 October 2001 / Accepted 28 June 2002

\begin{abstract}
It is generally accepted that relativistic jet outflows power the nonthermal emission from active galactic nuclei (AGN). The composition of these jets - leptonic versus hadronic - is still under debate. We investigate the microphysical details of the conversion process of the kinetic energy in collimated relativistic pair outflows into radiation through interactions with the ambient interstellar medium. Viewed from the coordinate system comoving with the pair outflow, the interstellar protons and electrons represent a proton-electron beam propagating with relativistic speed in the pair plasma. We demonstrate that the beam excites both electrostatic and low-frequency magnetohydrodynamic Alfven-type waves via a two-stream instability in the pair background plasma, and we calculate the time evolution of the distribution functions of the beam particles and the generated plasma wave turbulence power spectra. For standard AGN jet outflow and environment parameters we show that the initial beam distributions of interstellar protons and electrons quickly relax to plateau-distributions in parallel momentum, transferring thereby one-half of the initial energy density of the beam particles to electric field fluctuations of the generated electrostatic turbulence. On considerably longer time scales, the plateaued interstellar electrons and protons will isotropise by their self-generated transverse turbulence and thus be picked-up in the outflow pair plasma. These longer time scales are also characteristic for the development of transverse hydromagnetic turbulence from the plateaued electrons and protons. This hydromagnetic turbulence upstream and downstream is crucial for diffusive shock acceleration to operate at external or internal shocks associated with pair outflows.
\end{abstract}

Key words. galaxies: active - galaxies: jets - gamma-rays: theory - plasmas - turbulence - instabilities

\section{Introduction}

The detection of intense medium-energy gamma radiation from over 60 blazar active gactic nuclei (hereafter abbreviated as AGNs) with the EGRET instrument on the Compton observatory (Hartman et al. 1999) and $\mathrm{TeV}$ gamma radiation from several BL-Lac AGNs (Pohl 2001) shows that nonthermal gamma-ray production is a significant dissipation mechanism of jet energy generated by black-hole accretion. Besides the modelling of the broadband nonthermal radiation, the composition of the jet plasma - i.e. electron-positron pair jets (leptonic jets) versus electron-proton jets (hadronic jets) - and the

Send offprint requests to: $\mathrm{R}$. Schlickeiser, e-mail: rsch@tp4.ruhr-uni-bochum.de acceleration of these jet particles to relativistic energies are main subjects of the current theoretical research.

Gamma radiation in leptonic models of broadband blazar emission is attributed to synchrotron self-Compton (Maraschi et al. 1992; Bloom \& Marscher 1996; Tavecchio et al. 1998) or external Compton (Dermer \& Schlickeiser 1993; Sikora et al. 1994; Böttcher et al. 1997; Dermer et al. 1997; Arbeiter et al. 2002) processes (see Böttcher 2002 and Sikora \& Madejski 2001 for recent reviews). In hadronic models, secondary photopairs and photomesons and secondary mesons are produced when energetic protons and ions interact either with ambient synchrotron photons (Mannheim \& Biermann 1992; Mannheim 1993), photons of the external field (Bednarek \& Protheroe 1999; Atoyan \& Dermer 2000) and/or ambient matter fields (Pohl \& Schlickeiser 2000). Observationally, future 
detection of high-energy neutrino emission correlated with high-energy photon emission (Schuster et al. 2002) will provide the ultimate test between the leptonic and hadronic jet models.

Most existing radiation models of AGN jets are very unspecific on the microphysical details of the conversion of the kinetic jet energy into energetic charged particles and subsequently into radiation. Without detailed discussion these models often assume that a significant fraction of the accreted kinetic energy is injected into nonthermal pairs and/or hadrons with power-law distribution functions. This efficient conversion is attributed to the scenario that the outflowing relativistic jet plasma has produced a relativistic shock with fully developed hydromagnetic turbulence in order to allow for efficient non-thermal diffusive particle acceleration at the collisionless shock fronts:

(1) This neglects the fact that it takes a finite time to build up the necessary turbulence in the two-stream multi-fluid system consisting of the relativistically moving jet plasma and the traversed interstellar or intergalactic hydrogen plasma.

(2) It is not clear from the beginning that the outflowing relativistic jet plasma will generate primarily (and enough) transverse magnetohydrodynamic turbulence, which is crucial for efficient particle deflections in the up- and downstream region of the shock waves. It is well conceivable that most of the kinetic blast wave energy is transferred to electrostatic plasma turbulence and not to transverse hydromagnetic turbulence.

(3) The properties of the generated plasma turbulence are decisive both for the formation and the nature of the developing collisionless shock waves. It is known from non-relativistic shock theory that the inclusion of the finite pressure and energy flux of the generated plasma turbulence in the Rankine-Hugoniot shock relations strongly modifies the standard fluid shock properties (Vainio \& Schlickeiser 1999, 2001; Lerche et al. 2000) and subsequently the energy spectrum of the accelerated nonthermal particles.

It is the purpose of this work to consider more thoroughly some of the microphysical details of the energy conversion in relativistic jet outflows. We consider the energisation of relativistic particles in the jet by interactions with the surrounding medium following the earlier work of three of us (Pohl \& Schlickeiser 2000; Pohl et al. 2002). There the AGN jet has been assumed to be a cloud of dense electron-proton plasma which moves relativistically through the electron-proton interstellar medium of the AGN host galaxy. The plasma cloud is assumed to have a cylindrical shape with thickness $d^{*}=$ $10^{13} d_{13}^{*} \mathrm{~cm}$, which is small compared to the radius $r^{*}=$ $10^{14} r_{14}^{*} \mathrm{~cm}$ of the cylinder (see Fig. 1 of Pohl \& Schlickeiser 2000 for a sketch of the assumed cloud geometry). It was shown that in such hadronic jets swept-up ambient matter is quickly isotropised in the jet cloud frame by a relativistic twostream instability, which provides relativistic particles in the jet cloud without invoking any acceleration process. Here and in the following the index $*$ indicates the values of physical quantities in the laboratory (AGN) frame; quantities not indexed are in the jet frame.

Here we study the leptonic variant of this jet outflow model, i.e. we model the outflowing jet cloud as a one-dimensional channeled outflow of thickness $d^{*}$ with relativistic bulk velocity $V$, consisting of pairs of electrons and positrons of density $n_{b}^{*}$ instead of electrons and protons. To avoid dramatic pair annihilation, the density of pairs must be limited, and we allow for a thermal pair distribution with nonrelativistic temperature $\Theta=k_{B} T_{\text {pair }} /\left(m_{\mathrm{e}} c^{2}\right)<<1$ in the jet rest frame. This beam of pairs propagates into the surrounding interstellar medium that consists of cold protons and electrons at rest of density $n_{i}^{*}$.

For mathematical convenience we assume that the outflow is directed parallel to a uniform background magnetic field. The assumption of the magnetic field directed along the ejecta velocity enormously facilitates the analytical treatment of the two-stream instability, but crucially depends on the location of the particle energisation with respect to the large-scale magnetic field and the confinement of the jet ejecta. In magnetohydrodynamic models of jets in accreting systems the poloidal magnetic field component is more strongly $\left(\propto R^{-2}\right)$ suppressed by the side expansion of the ejecta than the transverse magnetic field component $\left(\propto R^{-1}\right)$, so that our assumption will hold at close distances to the central object. Moreover, in the presence of a dominating transverse magnetic field it can be argued that the ambient medium will penetrate the ejecta by one Larmor radius only and the momentum exchange should be faster and more efficient than in the case of a dominating poloidal magnetic field considered here ${ }^{1}$.

Viewed from the coordinate system comoving with the pair outflow, the interstellar protons and electrons represent a proton-electron beam propagating with relativistic speed $-V$ antiparallel to the uniform magnetic field direction. Modifying the analysis of Pohl \& Schlickeiser (2000) and Pohl et al. (2002) for cold electron-proton outflows to finite temperature pair outflows, we demonstrate that the beam excites both electrostatic and low-frequency magnetohydrodynamic Alfventype waves via a two-stream instability in the pair background plasma. We study the time evolution of the beam particles, the generated plasma wave turbulence power spectra and discuss the radiation signatures of such systems.

\section{Basic equations}

\subsection{Outflow parameters}

In the comoving frame the total phase space distribution function of the plasma in the blast wave region at the start (time $t=$ $0)$ is

$$
\begin{aligned}
& f(p, t=0)=n_{i} f_{i}(p, \mu, t=0)+n_{b} f_{b}(p, \mu, t=0)= \\
& \frac{1}{2 \pi p^{2}} n_{i} \delta(\mu+1) \delta(p-P)+n_{b} T(p, \Theta)
\end{aligned}
$$

with the thermal pair distribution

$T(p, \Theta)=\left[4 \pi\left(m_{\mathrm{e}} c\right)^{3} \Theta K_{2}\left(\Theta^{-1}\right)\right]^{-1} \exp \left(-\frac{\sqrt{1+\frac{p^{2}}{m_{\mathrm{e}}^{2} c^{2}}}}{\Theta}\right)$

${ }^{1}$ We are grateful to the referee for noting this difference. 
and the dimensionless pair temperature

$\Theta \equiv \frac{k_{B} T_{\text {pair }}}{m_{\mathrm{e}} c^{2}}$

Here $\mu=p_{\|} / p$ is the cosine of the pitch-angle of the particles in the magnetic field $B$, and $P=\Gamma m V=m c \sqrt{\Gamma^{2}-1}$ where $\Gamma=1 / \sqrt{1-\left(V^{2} / c^{2}\right)}$. The number densities transform as $n_{i}=$ $\Gamma n_{i}^{*}, \quad n_{b}=n_{b}^{*} / \Gamma$.

Earlier radiation modelling of AGN jets (e.g. Dermer \& Schlickeiser 1993; Pohl \& Schlickeiser 2000) indicated that the following parameters are appropriate: the density of pairs in the jet cloud is of order $n_{b}^{*}=10^{10} n_{b, 10}^{*} \mathrm{~cm}^{-3}$, implying $n_{b}=10^{8} n_{b, 8}=10^{8} n_{b, 10}^{*} \Gamma_{2}^{-1} \mathrm{~cm}^{-3}$, and much larger than the interstellar gas density $n_{i}^{*}$. The initial Lorentz factor is of order $\Gamma=10^{2} \Gamma_{2}$ and the total number of pairs in the plasmoid is $N_{\text {tot }}=3 \times 10^{51}\left(r_{14}^{*}\right)^{2} d_{13}^{*} n_{b, 10}^{*}$, corresponding to a total kinetic energy of $E_{\text {tot }}=5 \times 10^{47} \Gamma_{2}\left(r_{14}^{*}\right)^{2} d_{13}^{*} n_{b, 10}^{*}$ erg.

The background magnetic field in the blast wave is parametrized by the equipartition parameter $\epsilon_{B}=0.1 \epsilon_{B,-1}$ through

$B=0.14\left(\epsilon_{B,-1} n_{b, 8} \Theta_{-4}\right)^{1 / 2}$ Gauss

for a non-relativistic initial normalised (co-moving) pair temperature $\Theta=10^{-4} \Theta_{-4}$. The plasma parameter of the pair plasma

$g_{\mathrm{p}}=7.3 \times 10^{-4} n_{b}^{1 / 2}\left[\frac{m_{\mathrm{e}} c^{2} \Theta}{k_{B}}\right]^{-3 / 2}=$

$1.6 \times 10^{-8}\left(n_{b, 10}^{*}\right)^{1 / 2} \Gamma_{2}^{-1 / 2} \Theta_{-4}^{-3 / 2}<<1$

is much smaller than unity, so that the kinetic plasma description is appropriate.

The beam distribution function $f_{i}$ in Eq. (1) is unstable and excites electrostatic and low-frequency transverse plasma waves. We want to calculate the time scales $t_{\mathrm{e}}$ and $t_{\mathrm{t}}$ it takes these plasma waves to relax the incoming interstellar protonelectron beam to a stable distribution with respect to both instabilities. Of particular interest is the timescale $t_{\mathrm{t}}$ for beam isotropisation: if this relaxation time is much smaller than the light travel time $d / c$ then an isotropic distribution of protons and electrons in the blast wave region is efficiently generated.

\subsection{Parallel plasma waves in hot pair plasmas}

We restrict our analysis here to parallel propagating electrostatic and low-frequency transverse plasma waves. Because

$n_{i} / n_{b}=\Gamma^{2} n_{i}^{*} / n_{b}^{*}=10^{-6} \Gamma_{2}^{2} n_{i}^{*} / n_{b, 10}^{*}<<1$,

the beam is weak. Therefore the contributions from the beam to the plasma wave dispersion relations of electrostatic waves and transverse waves at frequencies $\omega_{\mathrm{R}}$ well below the nonrelativistic electron gyrofrequency $\left(\left|\omega_{\mathrm{R}}\right|<<\left|\Omega_{\mathrm{e}}\right|\right)$ are perturbations to the longitudinal $\left(\mathfrak{R} \Lambda_{\mathrm{e}}=0\right)$ and transverse dispersion relations $\left(\mathfrak{R} \Lambda_{t}=0\right)$ in the thermal pair plasma. The properties of the longitudinal and transverse dispersion relations in a thermal pair plasma are derived in Appendix A.
From the real part of the longitudinal dispersion relation in the thermal pair plasma we obtain the electrostatic waves

$\omega_{\mathrm{R}}^{2}=2 \omega_{\mathrm{p}, \mathrm{e}}^{2}$

in the wavenumber range $|k| \leq 2 \omega_{\mathrm{p}, \mathrm{e}} /\left(c \Theta^{1 / 2}\right)$ as the only longitudinal plasma mode. These waves are not damped $\left(\omega_{I}=\right.$ $0)$ in the superluminal wavenumber range $|k|<\sqrt{2} \omega_{\mathrm{p}, \mathrm{e}} / c$, whereas in the subluminal wavenumber range $\sqrt{2} \omega_{\mathrm{p}, \mathrm{e}} / c \leq|k| \leq$ $2 \omega_{\mathrm{p}, \mathrm{e}} /\left(c \Theta^{1 / 2}\right)$ the damping rate is

$$
\begin{aligned}
\omega_{I}= & -\pi \operatorname{sgn}\left(\omega_{\mathrm{R}}\right) \frac{\omega_{\mathrm{p}, \mathrm{e}}^{4}}{c k\left[c^{2} k^{2}-2 \omega_{\mathrm{p}, \mathrm{e}}^{2}\right] \Theta K_{2}(1 / \Theta)} \\
& \times \exp \left[-\frac{c|k|}{\Theta \sqrt{c^{2} k^{2}-2 \omega_{\mathrm{p}, \mathrm{e}}^{2}}}\right] \\
& \times\left[1+\frac{2 \Theta}{c|k|} \sqrt{c^{2} k^{2}-2 \omega_{, e}^{2}}+\frac{2 \Theta\left(c^{2} k^{2}-2 \omega_{\mathrm{p}, \mathrm{e}}^{2}\right)}{c^{2} k^{2}}\right]
\end{aligned}
$$

where

$\omega_{\mathrm{p}, \mathrm{e}}=\sqrt{4 \pi e^{2} n_{b} / m_{\mathrm{e}}}=5.64 \times 10^{8}\left(n_{b, 10}^{*}\right)^{1 / 2} \Gamma_{2}^{-1 / 2} \mathrm{~Hz}$

is the electron plasma frequency.

Likewise, from the real part of the transverse dispersion relation in the thermal pair plasma we obtain the transverse dispersion relation

$\omega_{\mathrm{R}}^{2} \simeq \frac{V_{\mathrm{e}}^{2} \Omega_{\mathrm{e}}^{2} k^{2}}{\Omega_{\mathrm{e}}^{2}+V_{\mathrm{e}}^{2} k^{2}}$

where

$\begin{aligned} \Omega_{\mathrm{e}} & =\frac{e B}{m_{\mathrm{e}} c}=1.76 \times 10^{7}(B / \mathrm{Gauss}) \mathrm{Hz} \\ & =2.46 \times 10^{6}\left(\epsilon_{B,-1} n_{b, 8} \Theta_{-4}\right)^{1 / 2} \mathrm{~Hz}\end{aligned}$

denotes the absolute value of the nonrelativistic electron gyrofrequency and

$V_{\mathrm{e}}=\frac{\Omega_{\mathrm{e}} c}{\sqrt{2} \omega_{\mathrm{p}, \mathrm{e}}}=9.2 \times 10^{7}\left(\epsilon_{B,-1} \Theta_{-4}\right)^{1 / 2} \mathrm{~cm} \mathrm{~s}^{-1}$

is the Alfven speed in the pair plasma. In Eq. (10) we adopt the convention that positive values of $\omega_{\mathrm{R}}>0$ denote left-handed circularly polarized waves, and that negative values of $\omega_{R}<0$ denote right-handed circularly polarized waves.

For small wavenumbers $|k|<<k_{\mathrm{e}}$, where

$k_{\mathrm{e}} \equiv \Omega_{\mathrm{e}} / V_{\mathrm{e}}=2.7 \times 10^{-2} n_{b, 8}^{1 / 2} \mathrm{~cm}^{-1}$

is the inverse pair skin length, Eq. (10) reduces to

$\omega_{\mathrm{R}}^{2}=V_{\mathrm{e}}^{2} k^{2}$

whereas in the opposite case $|k|>>k_{\mathrm{e}}$ Eq. (10) approaches the pair-cyclotron wave limit $\omega_{\mathrm{R}}^{2} \simeq \Omega_{\mathrm{e}}^{2}$. For small wavenumbers $\left(|k|<<k_{\mathrm{e}}\right)$ we obtain for the pair Alfven waves the damping rate

$\omega_{I, A}(k) \simeq-\frac{\pi^{1 / 2}}{2} \frac{\Omega_{\mathrm{e}} V_{\mathrm{e}}}{v_{\text {th,e }}} \frac{k_{\mathrm{e}}}{|k|} \exp \left[-\left(\frac{V_{\mathrm{e}} k_{\mathrm{e}}}{v_{\text {th, }} k}\right)^{2}\right]$

where $v_{\text {th,e }}=\sqrt{2 \Theta} c=\sqrt{2 k_{B} T_{\mathrm{e}} / m_{\mathrm{e}}}$ is the non-relativistic thermal pair velocity. 


\subsection{Time evolution of the electrostatic instability}

Neglecting spatial dependencies, the time-dependent behaviour of the intensities of the excited electrostatic waves is given by

$\frac{\partial I_{\mathrm{e}}(k, t)}{\partial t}=2 \psi_{\mathrm{e}} I_{\mathrm{e}}(k, t)$,

where the growth rate $\psi_{\mathrm{e}}$ is (Schlickeiser 2002, Ch. 11.2)

$\psi_{\mathrm{e}}(k) \simeq \frac{2 \pi^{2}}{\omega_{\mathrm{R}}\left[\frac{\partial \mathfrak{R} \Lambda_{\mathrm{L}}}{\partial \omega_{\mathrm{R}}}\right]} \sum_{i=\mathrm{e}, \mathrm{p}} \omega_{\mathrm{p}, i}^{2} \int_{-\infty}^{\infty} \mathrm{d} p_{\|} \int_{0}^{\infty} \mathrm{d} p_{\perp} \frac{p_{\|} p_{\perp}}{\gamma}$

$\delta\left(\frac{k p_{\|}}{\gamma m_{i}}-\omega_{\mathrm{R}}\right) \frac{\partial f_{i}}{\partial p_{\|}}=\pi^{2} \sum_{i=\mathrm{e}, \mathrm{p}} \omega_{\mathrm{p}, i}^{2} \int_{-\infty}^{\infty} \mathrm{d} p_{\|}$

$\int_{0}^{\infty} \mathrm{d} p_{\perp} \frac{p_{\|} p_{\perp}}{\gamma} \delta\left(\frac{k p_{\|}}{\gamma m_{i}}-\omega_{\mathrm{R}}\right) \frac{\partial f_{i}}{\partial p_{\|}}$

with

$\omega_{\mathrm{p}, i}^{2}=\frac{4 \pi n_{i} e_{i}^{2}}{m_{i}}$

Note that the summation $i=\mathrm{e}, \mathrm{p}$ refers to the incoming beam electrons and protons. Equation (16) holds provided that the initial growth rate $\psi_{\mathrm{e}}(t=0)$ due to the beam particles is much larger than the Landau damping rate $\omega_{\mathrm{I}}$ in the thermal pair plasma which is verified in Appendix B.

We do not know precisely the wave spectrum at time $t=0$. What we do know is that one starts with a beam particle spectrum that is a $\delta$-function in momentum parallel to the ambient magnetic field and, once the instability stops, the particle spectrum must be a plateau in $p_{\|}$. Thus the instability rate at late times can be effectively calculated using the weak turbulence limit. At early times such is not the case and more care has to be taken (Pohl et al. 2002). However, our interest here centers on the late time evolution when the weak turbulence approximation is particularly appropriate.

Integrating Eq. (16) over time gives

$$
\begin{aligned}
& I_{\mathrm{e}}(k, t)-I_{\mathrm{e}}(k, t=0)=2 \pi^{2} \sum_{i=\mathrm{e}, \mathrm{p}} \omega_{\mathrm{p}, i}^{2} \int_{-\infty}^{\infty} \mathrm{d} p_{\|} \int_{0}^{\infty} \mathrm{d} p_{\perp} \\
& \frac{p_{\|} p_{\perp}}{\gamma} \delta\left(\frac{k p_{\|}}{\gamma m_{i}}-\omega_{\mathrm{R}}\right) \int_{0}^{t} \mathrm{~d} t^{\prime} I\left(k, t^{\prime}\right) \frac{\partial f_{i}}{\partial p_{\|}} .
\end{aligned}
$$

At the same time the phase space density of the beam particles evolves as

$\frac{\partial f_{i}}{\partial t}=\pi e_{i}^{2} \frac{\partial}{\partial p_{\|}}\left[\int_{-\infty}^{\infty} \mathrm{d} k I(k, t) \delta\left(\omega_{\mathrm{R}}-\frac{k p_{\|}}{\gamma m_{i}}\right) \frac{\partial f_{i}}{\partial p_{\|}}\right]$.

In terms of the phase space distribution function

$f_{i}=\frac{1}{2 \pi p_{\perp}} \delta\left(p_{\perp}\right) G_{i}\left(p_{\|}, t\right)$ the two coupled Eqs. (19) and (20) read

$$
\begin{aligned}
& I_{\mathrm{e}}(k, t)-I_{\mathrm{e}}(k, t=0)=\pi \sum_{i=\mathrm{e}, \mathrm{p}} \omega_{\mathrm{p}, i}^{2} \int_{-\infty}^{\infty} \mathrm{d} p_{\|} \frac{p_{\|}}{\sqrt{1+\left(\frac{p_{\|}}{m_{i} c}\right)^{2}}} \\
& \delta\left(\frac{k p_{\|}}{m_{i} \sqrt{1+\left(\frac{p_{\|}}{m_{i} c}\right)^{2}}}-\omega_{\mathrm{R}}\right) \int_{0}^{t} \mathrm{~d} t^{\prime} I\left(k, t^{\prime}\right) \frac{\partial G_{i}\left(p_{\|}, t^{\prime}\right)}{\partial p_{\|}}
\end{aligned}
$$

and

$$
\begin{aligned}
& \frac{\partial G_{i}}{\partial t}=\pi e_{i}^{2} \frac{\partial}{\partial p_{\|}} \\
& {\left[\int_{-\infty}^{\infty} \mathrm{d} k I(k, t) \delta\left(\omega_{\mathrm{R}}-\frac{k p_{\|}}{m_{i} \sqrt{1+\left(\frac{p_{\|}}{m_{i}}\right)^{2}}}\right) \frac{\partial G_{i}}{\partial p_{\|}}\right] .}
\end{aligned}
$$

Performing the $k$-integral in Eq. (23) with

$\delta\left(\omega_{\mathrm{R}}-\frac{k p_{\|}}{m_{i} \sqrt{1+\left(\frac{p_{\|}}{m_{i} c}\right)^{2}}}\right)=$

$\frac{m_{i} \sqrt{1+\left(\frac{p_{\|}}{m_{i} c}\right)^{2}}}{\left|p_{\|}\right|} \delta\left(k-\frac{m_{i} \omega_{\mathrm{R}} \sqrt{1+\left(\frac{p_{\|}}{m_{i} c}\right)^{2}}}{p_{\|}}\right)$

yields

$\frac{\partial G_{i}}{\partial t}=\pi e_{i}^{2} m_{i} \frac{\partial}{\partial p_{\|}}\left[\frac{\sqrt{1+\left(\frac{p_{\|}}{m_{i} c}\right)^{2}}}{\left|p_{\|}\right|}\right.$

$\left.I\left(\frac{m_{i} \omega_{\mathrm{R}} \sqrt{1+\left(\frac{p_{\|}}{m_{i} c}\right)^{2}}}{p_{\|}}, t\right) \frac{\partial G_{i}}{\partial p_{\|}}\right]$

which can be integrated over $t$ and $p_{\|}$, resulting in

$$
\begin{aligned}
& \int_{0}^{p} \mathrm{~d} p_{\|}\left[G\left(p_{\|}, t\right)-G\left(p_{\|}, t=0\right)\right]=\pi e_{i}^{2} m_{i} \frac{\sqrt{1+\left(\frac{p}{m_{i} c}\right)^{2}}}{|p|} \\
& \int_{0}^{t} \mathrm{~d} t^{\prime} I\left(\frac{m_{i} \omega_{\mathrm{R}} \sqrt{1+\left(\frac{p}{m_{i} c}\right)^{2}}}{p}, t^{\prime}\right) \frac{\partial G_{i}\left(p, t^{\prime}\right)}{\partial p} .
\end{aligned}
$$

We then obtain

$$
\int_{0}^{t} \mathrm{~d} t^{\prime} I\left(\frac{m_{i} \omega_{\mathrm{R}} \sqrt{1+\left(\frac{p}{m_{i} c}\right)^{2}}}{p}, t^{\prime}\right) \frac{\partial G_{i}\left(p, t^{\prime}\right)}{\partial p}=
$$

$\frac{|p|}{\pi e_{i}^{2} m_{i} \sqrt{1+\left(\frac{p}{m_{i} c}\right)^{2}}} \int_{0}^{p} \mathrm{~d} p_{\|}\left[G\left(p_{\|}, t\right)-G\left(p_{\|}, t=0\right)\right]$. 
Likewise, we perform the $p_{\|}$-integration in the wave Eq. (22) using

$$
\begin{aligned}
& \delta\left(\frac{k p_{\|}}{m_{i} \sqrt{1+\left(\frac{p_{\|}}{m_{i} c}\right)^{2}}}-\omega_{\mathrm{R}}\right)= \\
& \frac{m_{i}}{|k|}\left(\frac{N^{2}}{N^{2}-1}\right)^{3 / 2} \delta\left(p_{\|}-\frac{m_{i} c \operatorname{sgn}(N)}{\sqrt{N^{2}-1}}\right)
\end{aligned}
$$

where $N=c k / \omega_{\mathrm{R}}$ is the index of refraction. We obtain

$$
\begin{aligned}
& I_{\mathrm{e}}(k, t)-I_{\mathrm{e}}(k, t=0)=\pi c \sum_{i=\mathrm{e}, \mathrm{p}} \frac{\omega_{\mathrm{p}, i}^{2} m_{i}^{2}}{|k|} \frac{N^{2} \operatorname{sgn}(N)}{\left(N^{2}-1\right)^{3 / 2}} \\
& \int_{0}^{t} \mathrm{~d} t^{\prime} I\left(k, t^{\prime}\right) \frac{\partial G_{i}\left(p_{\|}, t^{\prime}\right)}{\partial p_{\|}} \mid p_{\|}=\frac{m_{i} c \operatorname{sgn}(N)}{\sqrt{N^{2}-1}} .
\end{aligned}
$$

We note that precisely the left-hand side of Eq. (27) taken at the values of $p=\frac{m_{i} c \operatorname{sgn}(N)}{\sqrt{N^{2}-1}}$ appears in the wave Eq. (29), so that after inserting Eq. (27) we derive

$$
\begin{aligned}
& I_{\mathrm{e}}(k, t)-I_{\mathrm{e}}(k, t=0)=c^{2} \frac{|N| \operatorname{sgn}(N)}{|k|\left(N^{2}-1\right)^{3 / 2}} \sum_{i=\mathrm{e}, \mathrm{p}} \frac{\omega_{\mathrm{p}, i}^{2} m_{i}^{2}}{e_{i}^{2}} \\
& \int_{0}^{m_{i} \operatorname{csgn}(N) / \sqrt{N^{2}-1}} \mathrm{~d} p_{\|}\left[G\left(p_{\|}, t\right)-G\left(p_{\|}, t=0\right)\right]
\end{aligned}
$$

which relates electrostatic wave spectra and particle distribution functions at time $t$ to the respective quantities at time $t=0$. Before discussing the consequences of the quasilinear integral (30) for electrostatic waves we derive the corresponding quasilinear integral for transverse waves.

\subsection{Time evolution of the transverse instability}

Neglecting again spatial dependencies, the time-dependent behaviour of the intensities of the excited transverse waves is given by (Lee \& Ip 1987; Pohl \& Schlickeiser 2000)

$\frac{\partial I_{n}}{\partial t}=2 \psi_{n} I_{n}$,

where the growth rate $\psi_{n}$ is

$$
\begin{aligned}
& \psi_{n}(k) \simeq \mathfrak{I} \frac{\pi}{\omega_{\mathrm{R}}\left[\frac{\partial \mathscr{R} \Lambda_{\mathrm{T}}}{\partial \omega_{\mathrm{R}}}\right]} \sum_{i=\mathrm{e}, \mathrm{p}} \omega_{\mathrm{p}, i}^{2} \int_{-\infty}^{\infty} \mathrm{d} p_{\|} \int_{0}^{\infty} \mathrm{d} p_{\perp} \\
& \frac{p_{\perp}^{2}}{\gamma\left(\frac{k p_{\|}}{\gamma m_{i}}+\Omega_{i} \gamma^{-1}-\omega_{\mathrm{R}}\right)}\left[\frac{\partial f_{i}}{\partial p_{\perp}}+\frac{k}{\gamma m_{i} \omega_{\mathrm{R}}}\left(p_{\perp} \frac{\partial f_{i}}{\partial p_{\|}}-p_{\|} \frac{\partial f_{i}}{\partial p_{\perp}}\right)\right] \\
& =\frac{\pi^{2}}{\omega_{\mathrm{R}}\left[\frac{\partial \mathfrak{R} \Lambda_{\mathrm{T}}}{\partial \omega_{\mathrm{R}}}\right]} \sum_{i=\mathrm{e}, \mathrm{p}} \omega_{\mathrm{p}, i}^{2} \int_{-\infty}^{\infty} \mathrm{d} p_{\|} \int_{0}^{\infty} \mathrm{d} p_{\perp} \frac{p_{\perp}^{2}}{\gamma} \delta\left(\frac{k p_{\|}}{\gamma m_{i}}\right. \\
& \left.+\frac{\Omega_{i}}{\gamma}-\omega_{\mathrm{R}}\right)\left[\frac{\partial f_{i}}{\partial p_{\perp}}+\frac{k}{\gamma m_{i} \omega_{\mathrm{R}}}\left(p_{\perp} \frac{\partial f_{i}}{\partial p_{\|}}-p_{\|} \frac{\partial f_{i}}{\partial p_{\perp}}\right)\right] .
\end{aligned}
$$

Equation (31) holds provided that the initial growth rate $\psi_{n}(t=$ $0)$ due to the beam particles is much larger than the cyclotron damping rate $\omega_{\mathrm{I}, \mathrm{A}}$ of the pair Alfven waves in the thermal background pair plasma which is verified in Appendix B.

Again, we operate in the weak turbulence limit so that the long-time behaviour of the wave intensity can be used because, once again, we do not know the early time evolution of the wave spectrum.

Transforming from the momentum variables $\left(p_{\perp}, p_{\|}\right)$to the new variables

$y=\frac{p_{\|}}{m_{i} c}, \quad E=\gamma=\sqrt{1+\frac{p_{\|}^{2}+p_{\perp}^{2}}{\left(m_{i} c\right)^{2}}}$

we obtain

$\psi_{n}=\frac{\pi^{2}}{|k| c \omega_{\mathrm{R}}\left[\frac{\partial \mathcal{R} \Lambda_{\mathrm{T}}}{\partial \omega_{\mathrm{R}}}\right]} \sum_{i=\mathrm{e}, \mathrm{p}} \omega_{\mathrm{p}, i}^{2}\left(m_{i} c\right)^{3} \int_{1}^{\infty} \mathrm{d} E$

$\int_{-\sqrt{E^{2}-1}}^{\sqrt{E^{2}-1}} \mathrm{~d} y\left(E^{2}-1-y^{2}\right) \delta\left(y-\frac{E}{N}+x_{i}\right)\left(\frac{\partial f_{i}}{\partial E}+N \frac{\partial f_{i}}{\partial y}\right)$

where

$x_{i}=\frac{\Omega_{i}}{k c}$.

For Alfven speeds $V_{\mathrm{e}}$ that are much less than the speed of light $c$, the index of refraction of $N=c / V_{\mathrm{e}}>>1$ is large compared to unity, so that the derivative $\partial f_{i} / \partial E$ is negligible compared to $N\left(\partial f_{i} / \partial y\right)$, leading to

$$
\begin{aligned}
& \psi_{n}=\frac{\pi^{2} \operatorname{sgn}(k)}{\omega_{\mathrm{R}}^{2}\left[\frac{\partial \mathcal{R} \Lambda_{\mathrm{t}}}{\partial \omega_{\mathrm{R}}}\right]} \sum_{i=\mathrm{e}, \mathrm{p}} \omega_{\mathrm{p}, i}^{2}\left(m_{i} c\right)^{3} \\
& \int_{1}^{\infty} \mathrm{d} E \int_{-\sqrt{E^{2}-1}}^{\sqrt{E^{2}-1}} \mathrm{~d} y\left(E^{2}-1-y^{2}\right) \delta\left(y-\frac{E}{N}+x_{i}\right) \frac{\partial f_{i}}{\partial y} .
\end{aligned}
$$

Transforming from the variable $y$ to the cosine of the pitch angle $\mu=y / \sqrt{E^{2}-1}$ gives

$\psi_{n}=\frac{\pi^{2} \operatorname{sgn}(k)}{\omega_{\mathrm{R}}^{2}\left[\frac{\partial \mathcal{R} \Lambda_{\mathrm{t}}}{\partial \omega_{\mathrm{R}}}\right]} \sum_{i=\mathrm{e}, \mathrm{p}} \omega_{\mathrm{p}, i}^{2}\left(m_{i} c\right)^{3}$

$\int_{1}^{\infty} \mathrm{d} E \sqrt{E^{2}-1} \int_{-1}^{1} \mathrm{~d} \mu\left(1-\mu^{2}\right) \delta\left(\mu-\mu_{0}\right) \frac{\partial f_{i}}{\partial \mu}$

where

$\mu_{0}=\frac{E N^{-1}-x_{i}}{\sqrt{E^{2}-1}}$.

The $\mu$-integration gives a nonvanishing value provided $\left|\mu_{0}\right| \leq 1$ which is equivalent to the requirement $E \geq E_{i}$ where

$E_{i}=\frac{\sqrt{1-N^{-2}+x_{i}^{2}}-x_{i} N^{-1}}{1-N^{-2}} \simeq \sqrt{1+x_{i}^{2}}$ 
where the latter approximation holds in the limit $|N|>>1$. where

Consequently, we derive

$\psi_{n}=\frac{\pi^{2} \operatorname{sgn}(k)}{\omega_{\mathrm{R}}^{2}\left[\frac{\partial \mathfrak{R} \Lambda_{\mathrm{t}}}{\partial \omega_{\mathrm{R}}}\right]} \sum_{i=\mathrm{e}, \mathrm{p}} \omega_{\mathrm{p}, i}^{2}\left(m_{i} c\right)^{3}$

$\left.\int_{E_{i}}^{\infty} \mathrm{d} E \sqrt{E^{2}-1}\left(1-\mu_{0}^{2}\right) \frac{\partial f_{i}}{\partial \mu}\right|_{\mu=\mu_{0}}$.

In terms of the normalized phase space distribution function

$f_{i}=\delta(E-\Gamma) F_{i}(\mu, t) /\left[2 \pi\left(m_{i} c\right)^{3} \Gamma\left(\Gamma^{2}-1\right)^{1 / 2}\right]$

Eq. (40) reads

$\psi_{n}=\left.\frac{\pi \operatorname{sgn}(k)}{2 \omega_{\mathrm{R}}^{2}\left[\frac{\partial \mathfrak{R} \Lambda_{\mathrm{T}}}{\partial \omega_{\mathrm{R}}}\right]} \sum_{i=\mathrm{e}, \mathrm{p}} \frac{\omega_{\mathrm{p}, i}^{2}}{\Gamma} H\left[|k|-R_{i}^{-1}\right]\left[1-\mu_{i}^{2}\right] \frac{\partial F_{i}}{\partial \mu}\right|_{\mu=\mu_{i}}$

where

$\mu_{i}=\frac{\Gamma N^{-1}-x_{i}}{\sqrt{\Gamma^{2}-1}}=\frac{1}{k V}\left(\omega_{\mathrm{R}}-\frac{\Omega_{i}}{\Gamma}\right)$

$H$ denotes the step function, $R_{i}=V \Gamma /\left|\Omega_{i}\right|$ the gyroradius.

The assumption of very large $N=c k / \omega_{\mathrm{R}} \rightarrow \infty$, made before, corresponds formally to $\omega_{\mathrm{R}} \rightarrow 0$, so that we can approximate Eq. (43) as

$\mu_{i} \simeq \mu_{\mathrm{c}}=-\frac{\Omega_{i}}{\Gamma k V}=-\frac{\operatorname{sgn}\left(q_{i}\right)}{R_{i} k}$.

According to Appendix A

$\omega_{\mathrm{R}}^{2}\left[\frac{\partial \Re \Lambda_{\mathrm{T}}}{\partial \omega_{\mathrm{R}}}\right]=\frac{2 c^{2} k^{2}}{\omega_{\mathrm{R}}}+\frac{4 \omega_{\mathrm{p}, \mathrm{e}}^{2} \omega_{\mathrm{R}}^{3}}{\left(\omega_{\mathrm{R}}^{2}-\Omega_{\mathrm{e}}^{2}\right)^{2}} \simeq \frac{2 c^{2} k^{2}}{\omega_{\mathrm{R}}}$

for frequencies much less than the electron gyrofrequency. Inserting Eq. (45) in Eq. (42) we obtain

$\psi_{n}=\left.\frac{\pi \omega_{\mathrm{R}}}{4 k|k| c^{2}} \sum_{i=\mathrm{e}, \mathrm{p}} \frac{\omega_{\mathrm{p}, i}^{2}}{\Gamma} H\left[|k|-R_{i}^{-1}\right]\left[1-\mu_{\mathrm{c}}^{2}\right] \frac{\partial F_{i}}{\partial \mu}\right|_{\mu=\mu_{\mathrm{c}}}$.

With the dispersion relation (151) we find for forward (+) (i.e. $\omega_{\mathrm{R}}=V_{\mathrm{e}} k$ ) and backward (-) (i.e. $\omega_{\mathrm{R}}=-V_{\mathrm{e}} k$ ) Alfven waves that the wave growth rates are

$\psi_{ \pm}= \pm \psi$

with

$\psi=\left.\frac{\pi V_{\mathrm{e}}}{4|k| c^{2} \Gamma} \sum_{i=\mathrm{e}, i} \omega_{\mathrm{p}, i}^{2} H\left[|k|-R_{i}^{-1}\right]\left[1-\mu_{\mathrm{c}}^{2}\right] \frac{\partial F_{i}}{\partial \mu}\right|_{\mu=\mu_{\mathrm{c}}}$

and accordingly for the time evolution of the respective wave intensities (see Eq. (31))

$\frac{\partial I_{+}}{\partial t}=+2 \psi I_{+}, \quad \frac{\partial I_{-}}{\partial t}=-2 \psi I_{-}$.

The two Eqs. (49) yield the integrals

$I_{+}(t) I_{-}(t)=I_{+}(t=0) I_{-}(t=0)$

and

$\left[I_{+}(t)-I_{-}(t)\right]-\left[I_{+}(t=0)-I_{-}(t=0)\right]=Z(k)$

$$
\begin{gathered}
Z(k)=\frac{\pi V_{\mathrm{e}}}{2|k| c^{2} \Gamma} \sum_{i=\mathrm{e}, \mathrm{p}} \omega_{\mathrm{p}, i}^{2} H\left[|k|-R_{i}^{-1}\right] \\
\left.\sum_{n=+,-} \int_{0}^{t} \mathrm{~d} t^{\prime}\left[1-\mu_{\mathrm{c}}^{2}\right] \frac{\partial F_{i}}{\partial \mu}\right|_{\mu=\mu_{\mathrm{c}}} I_{n}\left(k, t^{\prime}\right) .
\end{gathered}
$$

The influence of these excited waves on the beam particles is described by the quasilinear Fokker-Planck equation for the resonant wave-particle interaction. For Alfvén waves the index of refraction is large compared to unity, so that the Lorentz force associated with the magnetic field of the waves is much stronger than the Lorentz force associated with the electric field. As a consequence, on the shortest time scale these waves scatter the particles in pitch angle $\mu$ but conserve their energy, i.e. the waves isotropise the beam particles. The Fokker-Planck equation for the evolution of the phase space density is then

$\frac{\partial F_{i}}{\partial t}=\frac{\partial}{\partial \mu}\left[D_{\mu \mu} \frac{\partial F_{i}}{\partial \mu}\right]$

where the pitch angle Fokker-Planck coefficient is determined by the two wave intensities

$D_{\mu \mu}=\sum_{n=+,-} \frac{\pi \Omega_{i}^{2}\left(1-\mu^{2}\right)}{2 B^{2} \Gamma^{2}}$

$\int_{-\infty}^{\infty} \mathrm{d} k I_{n}(k, t) \delta\left(\omega_{\mathrm{R}}-k V \mu-\frac{\Omega_{i}}{\Gamma}\right)$

$\simeq \sum_{n=+,-} \frac{\pi \Omega_{i}^{2}\left(1-\mu^{2}\right)}{2 B^{2} \Gamma^{2}} \int_{-\infty}^{\infty} \mathrm{d} k I_{n}(k, t) \delta\left(k V \mu+\frac{\Omega_{i}}{\Gamma}\right)$

where we again used the limit $\omega_{\mathrm{R}} \rightarrow 0$. Integrating Eq. (53) over pitch angle and time, and using Eq. (44) we find

$\int_{-1}^{\mu} \mathrm{d} \mu^{\prime}\left[F_{i}\left(\mu^{\prime}, t\right)-F_{i}\left(\mu^{\prime}, t=0\right)\right]=\frac{\pi \Omega_{i}^{2}}{2 \Gamma^{2} B^{2}} \sum_{n=+,-}\left(1-\mu^{2}\right)$

$\int_{-\infty}^{\infty} \mathrm{d} k \int_{0}^{t} \mathrm{~d} t^{\prime} I_{n}\left(k, t^{\prime}\right) \frac{\partial F_{i}}{\partial \mu} \delta\left(k V \mu+\frac{\Omega_{i}}{\Gamma}\right)=\frac{\pi \Omega_{i}^{2}}{2 B^{2} \Gamma^{2} V|\mu|}$

$\sum_{n=+,-} \int_{0}^{t} \mathrm{~d} t^{\prime}\left(1-\mu^{2}\right) I_{n}\left(-\frac{\Omega_{i}}{\Gamma V \mu}, t^{\prime}\right) \frac{\partial F_{i}}{\partial \mu}$

$=\frac{\pi \Omega_{i}^{2}}{2 B^{2} \Gamma^{2} V|\mu|} \sum_{n=+,-} \int_{0}^{t} \mathrm{~d} t^{\prime}\left(1-\mu^{2}\right) I_{n}\left(\frac{\mu_{\mathrm{c}} k}{\mu}, t^{\prime}\right) \frac{\partial F_{i}}{\partial \mu}$.

Evaluating Eq. (55) at $\mu=\mu_{\mathrm{c}}$ gives

$\left.\sum_{n=+,-} \int_{0}^{t} \mathrm{~d} t^{\prime} I_{n}\left(k, t^{\prime}\right)\left(1-\mu_{\mathrm{c}}^{2}\right) \frac{\partial F_{i}}{\partial \mu}\right|_{\mu=\mu_{\mathrm{c}}}=$

$\frac{2 \Gamma^{2} B^{2} V}{\pi \Omega_{i}^{2}}\left|\mu_{\mathrm{c}}\right| \int_{-1}^{\mu_{\mathrm{c}}} \mathrm{d} \mu^{\prime}\left[F_{i}\left(\mu^{\prime}, t\right)-F_{i}\left(\mu^{\prime}, t=0\right)\right]$ 
which can be inserted into Eq. (52) to yield

$Z(k)=\frac{V_{\mathrm{e}} B^{2}}{V|k| c^{2} \Gamma} \sum_{i=\mathrm{e}, \mathrm{p}} H\left[|k|-R_{i}^{-1}\right] \omega_{\mathrm{p}, i}^{2} R_{i}^{2}\left|\mu_{\mathrm{c}}\right|$

$\times \int_{-1}^{\mu_{\mathrm{c}}} \mathrm{d} \mu^{\prime}\left[F_{i}\left(\mu^{\prime}, t\right)-F_{i}\left(\mu^{\prime}, t=0\right)\right]$

$=\frac{V_{\mathrm{e}} B^{2}}{V k^{2} c^{2} \Gamma} \sum_{i=\mathrm{e}, \mathrm{p}} H\left[|k|-R_{i}^{-1}\right] \omega_{\mathrm{p}, i}^{2} R_{i}$

$\times \int_{-1}^{\mu_{\mathrm{c}}} \mathrm{d} \mu^{\prime}\left[F_{i}\left(\mu^{\prime}, t\right)-F_{i}\left(\mu^{\prime}, t=0\right)\right]$.

The system of Eqs. (50), (51), and (57) has the general solution at time $t$

$I_{+}(t)=\sqrt{Y+\frac{1}{4}\left(Z+I_{+}(0)-I_{-}(0)\right)^{2}}$

$+0.5\left(Z+I_{+}(0)-I_{-}(0)\right)$

and

$I_{-}(t)=\sqrt{Y+\frac{1}{4}\left(Z+I_{+}(0)-I_{-}(0)\right)^{2}}$

$-0.5\left(Z+I_{+}(0)-I_{-}(0)\right)$

where

$Y \equiv I_{+}(0) I_{-}(0)$

If the initial turbulence is much weaker than the self-generated turbulence $I(k, 0)<<|Z(k)|$ and has a vanishing cross-helicity $I_{+}(k, 0)=I_{-}(k, 0)=I(k, 0)$ we obtain for Eqs. $(58),(59)$ approximately

$I_{ \pm}(k, t) \simeq \frac{1}{2}[|Z| \pm Z]+\frac{2 I^{2}(k, 0)}{|Z|}$

which relates the transverse wave spectra and particle distribution functions at time $t$ to the respective quantities at time $t=0$.

\section{Self-excited turbulence and initial beam relaxation}

We first discuss which of the two, electrostatic or transverse, relaxation processes on the incoming interstellar electron-proton beam is faster. Hence we calculate the power spectra of the selfexcited electrostatic and transverse turbulence from the derived quasilinear integrals for the beam initial condition.

For both relaxation processes the initial boundary condition for the evolution is the same: in the beginning $(t=0)$ there is a mono-energetic beam distribution (1), i.e. in terms of the two normalised distributions (21) and (41)

$G_{\mathrm{p}, \mathrm{e}}\left(p_{\|}, t=0\right)=\delta\left(p_{\|}+P\right)$,

$F_{\mathrm{p}, \mathrm{e}}(\mu, t=0)=\delta(\mu+1)$.
The final state of the evolution of the electrostatic instability is reached at time $t_{\mathrm{e}}$ when both growth rate and temporal derivative of the particle distribution function are zero, i.e. when $\partial G_{i} / \partial p_{\|}=0$ which is referred to as the "plateau distribution"

$G_{i}\left(p_{\|}, t_{\mathrm{e}}\right)=\frac{1}{P} H\left[p_{\|}+P\right] H\left[-p_{\|}\right]$.

The final state of the evolution of the transverse instability is reached at time $t_{\mathrm{t}}$ when both growth rate and temporal derivative of the distribution are zero, i.e. when $\partial F_{i} / \partial \mu=0$. Consequently here

$F_{i}\left(\mu, t=t_{\mathrm{t}}\right)=\frac{1}{2}$.

At this time the magnetohydrodynamic waves have completely isotropised the beam distribution.

\subsection{Self-excited electrostatic turbulence}

Inserting Eqs. (62) and (63) in Eq. (30) allows us to integrate over $p_{\|}$to obtain

$$
\begin{aligned}
& \operatorname{sgn}(N) \int_{0}^{m_{i} c \operatorname{sgn}(N) / \sqrt{N^{2}-1}} d p_{\|}\left[G\left(p_{\|}, t_{\mathrm{e}}\right)-G\left(p_{\|}, t=0\right)\right] \\
& =\frac{H[-N]}{\sqrt{N^{2}-1} \sqrt{\Gamma^{2}-1}} H\left[\Gamma-\frac{|N|}{\sqrt{N^{2}-1}}\right]
\end{aligned}
$$

so that the fully developed electrostatic turbulence is

$I_{\mathrm{e}}\left(k, t_{\mathrm{e}}\right)-I_{\mathrm{e}}(k, t=0)=\frac{c^{2}}{\sqrt{\Gamma^{2}-1}} \frac{|N| H[-N]}{|k|\left(N^{2}-1\right)^{2}}$

$H\left[\Gamma-\frac{|N|}{\sqrt{N^{2}-1}}\right] \sum_{i=\mathrm{e}, \mathrm{p}} \frac{\omega_{\mathrm{p}, i}^{2} m_{i}^{2}}{e_{i}^{2}}$

$=\frac{4 \pi n_{i} m_{\mathrm{p}} c^{2}}{\sqrt{\Gamma^{2}-1}}\left(1+\frac{m_{\mathrm{e}}}{m_{\mathrm{p}}}\right) \frac{|N| H[-N]}{|k|\left(N^{2}-1\right)^{2}} H\left[\Gamma-\frac{|N|}{\sqrt{N^{2}-1}}\right]$.

The total enhancement in electric field fluctuation power due to the plateauing of the proton and electron distribution function is obtained by integrating Eq. (66)

$$
\begin{aligned}
& (\delta E)^{2}=\int_{-\infty}^{0} \mathrm{~d} k\left[I_{\mathrm{e}}\left(k, t_{\mathrm{e}}\right)-I_{\mathrm{e}}(k, t=0)\right] \\
& =\frac{4 \pi n_{i} m_{\mathrm{p}} c^{2}}{\sqrt{\Gamma^{2}-1}}\left(1+\frac{m_{\mathrm{e}}}{m_{\mathrm{p}}}\right) \int_{\left(1-\Gamma^{-2}\right)^{-1 / 2}}^{\infty} \frac{\mathrm{d} N}{\left(N^{2}-1\right)^{2}} \\
& =\frac{2 \pi n_{i} m_{\mathrm{p}} c^{2}}{\sqrt{\Gamma^{2}-1}}\left(1+\frac{m_{\mathrm{e}}}{m_{\mathrm{p}}}\right)\left[\Gamma \sqrt{\Gamma^{2}-1}-\frac{1}{2} \ln \frac{\Gamma+\sqrt{\Gamma^{2}-1}}{\Gamma-\sqrt{\Gamma^{2}-1}}\right] \\
& \simeq 2 \pi \Gamma n_{i} m_{\mathrm{p}} c^{2}\left(1+\frac{m_{\mathrm{e}}}{m_{\mathrm{p}}}\right)
\end{aligned}
$$

so that the change in the electric field fluctuation energy density is

$\Delta U_{(\delta E)}=\frac{(\delta E)^{2}}{8 \pi}=\frac{1}{4} \Gamma n_{i} m_{\mathrm{p}} c^{2}\left(1+\frac{m_{\mathrm{e}}}{m_{\mathrm{p}}}\right)$. 
Electrostatic waves possess equipartition between electric and velocity fluctuations. So that the total change in fluctuation energy density due to plateauing is

$\Delta U=2 \Delta U_{(\delta E)}=\frac{1}{2} \Gamma n_{i} m_{\mathrm{p}} c^{2}\left(1+\frac{m_{\mathrm{e}}}{m_{\mathrm{p}}}\right)$

which is half of the initial energy density of the beam particles.

\subsection{Electrostatic beam relaxation}

Using the fully developed turbulence spectrum (66) we obtain for the Fokker-Planck Eq. (25) in the variable $x=\left|p_{\|}\right| / m_{i} c$

$\frac{\partial G_{i}}{\partial t}=\frac{\partial}{\partial x}\left[\frac{x^{2}}{\tau_{i}(x)} \frac{\partial G_{i}}{\partial x}\right]$

with the time scale

$\tau_{i}(x)=\frac{\sqrt{2\left(\Gamma^{2}-1\right)}}{\pi\left(1+\frac{m_{\mathrm{e}}}{m_{\mathrm{p}}}\right)} \frac{m_{i}^{2}}{m_{\mathrm{e}} m_{\mathrm{p}}} \frac{n_{b}}{n_{i}} \omega_{\mathrm{p}, \mathrm{e}}^{-1} \frac{1}{x \sqrt{1+x^{2}}}$

for values of $0 \leq x \leq \sqrt{\Gamma^{2}-1}$.

Evaluating this time scale at the momentum $x=P / m_{i} c$ yields for the beam electrons

$t_{\mathrm{e}, \mathrm{e}}=\frac{\sqrt{2}}{\pi \omega_{\mathrm{p}, \mathrm{e}}} \frac{m_{\mathrm{e}}}{m_{\mathrm{p}}\left(1+\frac{m_{\mathrm{e}}}{m_{\mathrm{p}}}\right)} \frac{n_{b}}{n_{i} \Gamma}$

and for the beam protons

$t_{\mathrm{e}, \mathrm{p}}=\left(\frac{m_{\mathrm{p}}}{m_{\mathrm{e}}}\right)^{2} t_{\mathrm{e}, \mathrm{e}}$

\subsection{Self-excited transverse turbulence}

Inserting Eqs. (62) and (64) in Eq. (57) allows us to integrate over $\mu$ to obtain with

$\int_{-1}^{\mu_{\mathrm{c}}} \mathrm{d} \mu^{\prime}\left[F_{i}\left(\mu^{\prime}, t_{\mathrm{t}}\right)-F_{i}\left(\mu^{\prime}, t=0\right)\right]=-\frac{1}{2}\left[1-\mu_{\mathrm{c}}\right]$

so that

$Z(k)=-\frac{V_{\mathrm{e}} B^{2}}{2 V k^{2} c^{2} \Gamma} \sum_{i=\mathrm{e}, \mathrm{p}} H\left[|k|-R_{i}^{-1}\right] \omega_{\mathrm{p}, i}^{2} R_{i}\left[1+\frac{\operatorname{sgn}\left(q_{i}\right)}{R_{i} k}\right]$

$=-\frac{V_{\mathrm{e}} B^{2} R_{\mathrm{p}} \omega_{\mathrm{p}, \mathrm{e}}^{2}}{2 V k^{2} c^{2}} \frac{n_{i}}{n_{b} \Gamma} \frac{m_{\mathrm{e}}}{m_{\mathrm{p}}}\left(H\left[|k|-R_{\mathrm{p}}^{-1}\right]\left[1+\frac{1}{R_{\mathrm{p}} k}\right]+\right.$

$\left.H\left[|k|-\frac{m_{\mathrm{p}}}{m_{\mathrm{e}}} R_{\mathrm{p}}^{-1}\right]\left[1-\frac{\left(m_{\mathrm{p}} / m_{\mathrm{e}}\right)}{R_{\mathrm{p}} k}\right]\right)$

which is negative for all wavenumber values. According to Eq. (61) this implies that predominantly backward moving lefthanded and right-handed polarized Alfven waves are generated. If the initial turbulence is negligibly small the respective intensities of the fully developed turbulence are

$I_{-}\left(k, t_{\mathrm{t}}\right)=|Z(|k|)|, \quad I_{+}\left(k, t_{\mathrm{t}}\right)=0$.
The total enhancement in magnetic field fluctuation power due to the isotropisation of the proton and electron distribution function is obtained by integrating Eq. (51) using Eq. (76)

$$
\begin{aligned}
(\delta B)_{-}^{2} & =\int_{-\infty}^{\infty} \mathrm{d} k \sum_{n= \pm}\left[I_{n}\left(k, t_{\mathrm{t}}\right)-I_{n}(k, t=0)\right] \\
& =-\int_{-\infty}^{\infty} \mathrm{d} k Z(k)=\frac{m_{\mathrm{e}}}{m_{\mathrm{p}}} \frac{V_{\mathrm{e}}}{V} \frac{n_{i}}{n_{b} \Gamma} \frac{B^{2} R_{\mathrm{p}}^{2} \omega_{\mathrm{p}, \mathrm{e}}^{2}}{c^{2}}\left[1+\frac{m_{\mathrm{e}}}{m_{\mathrm{p}}}\right] \\
& =4 \pi n_{i} \Gamma m_{\mathrm{p}} V_{\mathrm{e}} V\left[1+\frac{m_{\mathrm{e}}}{m_{\mathrm{p}}}\right]
\end{aligned}
$$

so that the change in the magnetic field fluctuation energy density is

$\Delta U_{\delta B}=\frac{(\delta B)_{-}^{2}}{8 \pi}=\frac{1}{2} n_{i} m_{\mathrm{p}} V_{\mathrm{e}} V \Gamma\left[1+\frac{m_{\mathrm{e}}}{m_{\mathrm{p}}}\right]$.

Alfven waves possess equipartition of wave energy densities between magnetic and plasma velocity fluctuations, so that the total change in fluctuation energy density to pitch angle isotropisation is

$\Delta U=\Delta U_{\delta B}+\Delta U_{\delta v}=2 \Delta U_{\delta B}=n_{i} m_{\mathrm{p}} V_{\mathrm{e}} V \Gamma\left[1+\frac{m_{\mathrm{e}}}{m_{\mathrm{p}}}\right]$.

Following exactly the same calculation as in Pohl \& Schlickeiser (2000) one can demonstrate that the increase (79) in the energy density is balanced by a corresponding decrease in the energy density of the beam protons and electrons during their isotropisation. The transverse plasma turbulence is generated at the expense of the beam particles which relax to a state of lower energy density. The change in the energy density of the beam particles is given by $-\Delta U$ which is the fraction $V_{\mathrm{e}} / c$ of the initial beam energy density.

\subsection{Transverse beam relaxation}

We can estimate the isotropisation length of the beam particles by using the fully-developed turbulence spectrum of backward moving transverse waves (75)-(76)

$I_{-}\left(k, t_{\mathrm{t}}\right) \simeq i_{-}|k|^{-2} H\left[|k|-R_{\mathrm{p}}^{-1}\right]$

with

$i_{-}=\frac{V_{\mathrm{e}} B^{2} R_{\mathrm{p}} \omega_{\mathrm{p}, \mathrm{e}}^{2}}{2 V k^{2} c^{2}} \frac{n_{i}}{n_{b} \Gamma} \frac{m_{\mathrm{e}}}{m_{\mathrm{p}}}$

to calculate the pitch angle Fokker-Planck coefficient (54)

$D_{\mu \mu}=\frac{\pi \Omega_{i}^{2}\left(1-\mu^{2}\right)}{2 B^{2} \Gamma^{2}} \int_{-\infty}^{\infty} \mathrm{d} k I_{-}\left(k, t_{\mathrm{t}}\right) \delta\left(\omega_{\mathrm{R}}-k V \mu-\frac{\Omega_{i}}{\Gamma}\right)$

$=d_{i}\left(1-\mu^{2}\right) \int_{-\infty}^{\infty} \mathrm{d} k|k|^{-2} H\left[|k|-R_{\mathrm{p}}^{-1}\right] \delta\left(\omega_{\mathrm{R}}-k V \mu-\frac{\Omega_{i}}{\Gamma}\right)$

where

$d_{i}=\frac{\pi}{4} \frac{\Omega_{i}^{2}}{\Gamma^{2}} \frac{V_{\mathrm{e}} \omega_{\mathrm{p}, \mathrm{e}}^{2}}{\Omega_{\mathrm{p}} c^{2}} \frac{n_{i}}{n_{b}} \frac{m_{\mathrm{e}}}{m_{\mathrm{p}}}$. 
The cyclotron resonance condition of beam particles of species $i=\mathrm{e}, \mathrm{p}$ appearing in the argument of the $\delta$-function in Eq. (82)

$\omega_{\mathrm{R}}-k V \mu=\Omega_{i} / \Gamma$,

when combined with the pair-plasma dispersion relation (with $V_{\mathrm{e}}^{2} \ll c^{2}$ ) for backward moving waves,

$\omega_{\mathrm{R}}=-V_{\mathrm{e}} k \sqrt{\frac{\Omega_{\mathrm{e}}^{2}}{\Omega_{\mathrm{e}}^{2}+k^{2} V_{\mathrm{e}}^{2}}}$,

gives the particle pitch angle cosine as a function of the resonant wavenumber in the form

$\mu_{i}(k)=-\epsilon\left[\sqrt{\frac{\Omega_{\mathrm{e}}^{2}}{\Omega_{\mathrm{e}}^{2}+k^{2} V_{\mathrm{e}}^{2}}}+\frac{\Omega_{i}}{k V_{\mathrm{e}} \Gamma}\right]$

where we introduced

$\epsilon \equiv V_{\mathrm{e}} / V$

In our convention, positive [negative] $k$ corresponds to right [left] handed polarization.

It is convenient to introduce the dimensionless wavenumber $x=k / k_{\mathrm{e}}$ and

$\gamma_{i} \equiv \frac{\Gamma_{i}\left|\Omega_{\mathrm{e}}\right|}{\Omega_{i}}$

so that

$D_{\mu \mu}=\frac{d_{i}}{V_{\mathrm{e}} k_{\mathrm{e}}^{2}}\left(1-\mu^{2}\right) \int_{-\infty}^{\infty} \mathrm{d} x x^{-2} H\left[|x|-\epsilon \gamma_{\mathrm{p}}^{-1}\right] \delta\left(x g_{i}(x)\right)$

with

$g_{i}(\mu, x)=\frac{\mu}{\epsilon}+\left(1+x^{2}\right)^{-1 / 2}+\left(\gamma_{i} x\right)^{-1}$.

In terms of the dimensionless notation the resonance condition (84) then is equivalent to $g_{i}(\mu, x)=0$ yielding

$\frac{\mu(x)}{\epsilon}=-\left[\left(1+x^{2}\right)^{-1 / 2}+\left(\gamma_{i} x\right)^{-1}\right]$.

As a consequence of pitch-angle scattering the beam particles adjust to the isotropic distribution on a length scale given by the scattering length

$\lambda=\frac{3 V}{8} \int_{-1}^{1} \mathrm{~d} \mu \frac{\left(1-\mu^{2}\right)^{2}}{D_{\mu \mu}(\mu)}$

Eq. (92) is valid for scattering lengths $\lambda$ larger than the gyroradius of the particles, $R_{\mathrm{p}}=3 \times 10^{9} \Gamma_{3} / B \mathrm{~cm}$ and $R_{\mathrm{e}}=1.7 \times$ $10^{6} \Gamma_{3} / B \mathrm{~cm}$ for protons and electrons, respectively. If the thickness $d$ of the outflow region is larger than the scattering length, indeed an isotropic distribution of the inflowing interstellar protons and electrons with Lorentzfactor $\langle\Gamma\rangle=\Gamma\left(1-\frac{V_{\mathrm{e}} V}{c^{2}}\right) \simeq \Gamma$ in the blast wave frame is efficiently generated.

From the scattering length we derive the isotropisation time scale in the outflow plasma

$t_{\mathrm{t}}=\lambda / c$

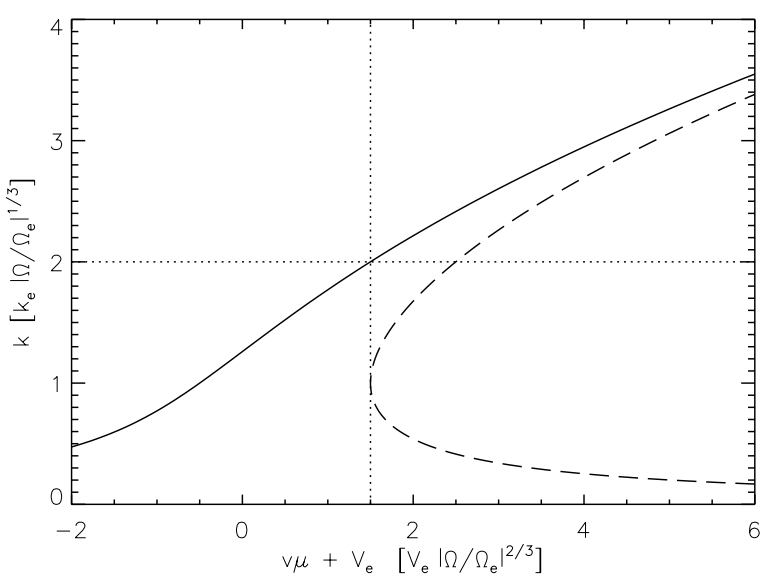

Fig. 1. Resonant wavenumber as a function of parallel momentum near the Alfvénic resonance gap. Here, $\Omega=\Omega_{i} / \Gamma, v=V$, the solid [dashed] curve gives $k$ for the right-handed [left-handed] waves for ions and vice-versa for electrons.

\subsubsection{Beam proton cyclotron resonance in a pair plasma}

For beam protons

$\gamma_{\mathrm{p}}=m_{\mathrm{p}} \Gamma / m_{\mathrm{e}}>>1$

is large and positive. According to Eq. (91) for protons, $\mu_{\mathrm{p}}(x)$ is a monotonically increasing function for $x>0$ going from $-\infty$ to 0 as $x$ goes from 0 to $\infty$. For $x<0, \mu_{\mathrm{p}} \rightarrow \infty$ as $x \rightarrow 0-$, but as $-x$ increases, $\mu_{\mathrm{p}}$ has a minimum at

$x_{\mathrm{E}}=-\gamma_{\mathrm{p}}^{-1 / 3}\left[1-\gamma_{\mathrm{p}}^{-2 / 3}\right]^{-1 / 2} \simeq-\gamma_{\mathrm{p}}^{-1 / 3}$

and $\mu_{\mathrm{p}}=0$ at

$x_{0}=-\left(\gamma_{\mathrm{p}}^{2}-1\right)^{-1 / 2} \simeq-\gamma_{\mathrm{p}}^{-1}$.

The minimum value is

$\mu_{\mathrm{E}}=-\epsilon\left[1-\gamma_{\mathrm{p}}^{-2 / 3}\right]^{3 / 2} \simeq-\epsilon\left[1-\frac{3}{2} \gamma_{\mathrm{p}}^{-2 / 3}\right] \simeq-\epsilon$.

Because this value is negative, there is no resonance gap for protons in a pair plasma even in a case of degenerate cross helicity. This is in contrast with the situation in a cold electronproton plasma, where one has to rely on electron-cyclotron waves scattering ions if waves propagating in one direction only are considered (see Vainio 2000 for a complete analysis of the resonance conditions in that case).

The $x$-value of the right-handed wave in resonance with the extreme value $\mu_{\mathrm{E}}$ achievable by the left-handed waves is approximately

$x \simeq 2 \gamma_{\mathrm{p}}^{-1 / 3}$

which is always much less than unity, indicating that we are well in the non-dispersive range of the dispersion relation of the right-handed waves. Thus, as far as phase speeds are considered, we can regard all the resonant waves as Alfvénic. This is because dispersion modifies the resonance conditions enabling relativistic particles to interact with the Alfven waves over the whole momentum space. We have plotted the resonant wavenumber as a function of $V \mu$ close to $V \mu=-V_{\mathrm{e}}$ in Fig. 1 . The figure is plotted for protons with $\Gamma=100$. 


\subsubsection{Beam electron cyclotron resonance in a pair plasma}

For relativistic beam electrons $\left(\gamma_{\mathrm{e}}=-\Gamma_{\mathrm{e}}=-\Gamma<0\right.$ is a large negative number), the analysis is very similar. The only difference is that $x$ has to change sign relative to the proton case. Now one has a monotonic resonance function, $\mu_{\mathrm{e}}(x)$, with the lefthanded waves, and the turning point in $\mu_{\mathrm{e}}(x)$ for right-handed waves. $\mu_{\mathrm{e}}$ has a minimum at

$x_{\mathrm{E}}=\Gamma_{\mathrm{e}}^{-1 / 3}\left[1-\Gamma_{\mathrm{e}}^{-2 / 3}\right]^{-1 / 2} \simeq \Gamma_{\mathrm{e}}^{-1 / 3}$

and $\mu_{\mathrm{e}}=0$ at

$x_{0}=\left(\Gamma_{\mathrm{e}}^{2}-1\right)^{-1 / 2} \simeq \Gamma_{\mathrm{e}}^{-1}$.

The minimum value is

$\mu_{\mathrm{E}}=-\epsilon\left[1-\Gamma_{\mathrm{e}}^{-2 / 3}\right]^{3 / 2} \simeq-\epsilon\left[1-\frac{3}{2} \Gamma_{\mathrm{e}}^{-2 / 3}\right] \simeq-\epsilon$.

Having established that no resonance gaps exist in the quasilinear interaction of the particles and the backward moving waves of relatively low wavenumbers, we will assume that other wave modes do not affect the dynamics of the system. In this case we can evaluate the wave spectra also with an alternative bispherical shell method which is described in Appendix A.

\subsubsection{Scattering length of beam protons}

The function $g_{i}(\mu, x)$ appearing in the Fokker-Planck coefficient (89) for beam protons is

$g_{\mathrm{p}}(\mu, x)=\frac{\mu}{\epsilon}+\left(1+x^{2}\right)^{-1 / 2}+\left(\gamma_{\mathrm{p}} x\right)^{-1}$.

According to the discussion of the proton cyclotron resonance condition we approximate the function $g_{\mathrm{p}}(\mu, x)$ as

$g_{\mathrm{p}}(\mu, x) \simeq \begin{cases}\frac{\mu}{\epsilon}+\frac{1}{x \gamma_{\mathrm{p}}} & \text { for }-1 \leq \mu \leq-\epsilon \\ \frac{\mu}{\epsilon}-\frac{\gamma_{\mathrm{p}} x+1}{\gamma_{\mathrm{p}}^{2 / 3}-1} & \text { for }-\epsilon \leq \mu \leq 0 \\ \frac{\mu}{\epsilon}+1+\frac{1}{\gamma_{\mathrm{p}} x} & \text { for } 0 \leq \mu \leq 1\end{cases}$

allowing a straightforward approximation of the proton FokkerPlanck coefficient

$D_{\mu \mu}^{\mathrm{p}}(\mu) \simeq \frac{d_{\mathrm{p}} \gamma_{\mathrm{p}}^{2}\left(1-\mu^{2}\right)}{V_{\mathrm{e}} k_{\mathrm{e}}^{2}}$

$\begin{cases}\frac{|\mu|}{\epsilon} & \text { for }-1 \leq \mu \leq-\epsilon \\ \left(\gamma_{\mathrm{p}}^{2 / 3}-1\right)\left|\left(\gamma_{\mathrm{p}}^{2 / 3}-1\right) \frac{\mu}{\epsilon}-1\right|^{-3} & \text { for }-\epsilon \leq \mu \leq 0 \\ \left|\frac{\mu}{\epsilon}+1\right| & \text { for } 0 \leq \mu \leq 1 .\end{cases}$

Inserting Eq. (104) into Eq. (92) gives the proton scattering length

$$
\begin{aligned}
\lambda_{\mathrm{p}}= & \frac{3 V V_{\mathrm{e}} k_{\mathrm{e}}^{2}}{8 d_{\mathrm{p}} \gamma_{\mathrm{p}}^{2}}\left[\epsilon \int_{-1}^{-\epsilon} \mathrm{d} \mu \frac{\left(1-\mu^{2}\right)}{|\mu|}\right. \\
& +\frac{1}{\gamma_{\mathrm{p}}^{2 / 3}-1} \int_{-\epsilon}^{0} \mathrm{~d} \mu\left(1-\mu^{2}\right)\left|\left(\gamma_{\mathrm{p}}^{2 / 3}-1\right) \frac{\mu}{\epsilon}-1\right|^{3} \\
& \left.+\int_{0}^{1} \mathrm{~d} \mu \frac{\left(1-\mu^{2}\right)}{\left|1+\frac{\mu}{\epsilon}\right|}\right] \simeq \frac{3 V_{\mathrm{e}}^{2} k_{\mathrm{e}}^{2}}{8 d_{\mathrm{p}} \gamma_{\mathrm{p}}^{2}}\left[2 \ln \epsilon^{-1}+\frac{\left(\gamma_{\mathrm{p}}^{2 / 3}-1\right)^{2}}{4}-1\right] \\
\simeq & \frac{3}{32} \frac{V_{\mathrm{e}}^{2} k_{\mathrm{e}}^{2}}{d_{\mathrm{p}} \gamma_{\mathrm{p}}^{2 / 3}}=\frac{3 \sqrt{2}}{8 \pi} \frac{c}{\omega_{\mathrm{p}, \mathrm{e}}} \frac{n_{b}}{n_{i}}\left(\frac{m_{\mathrm{p}} \Gamma}{m_{\mathrm{e}}}\right)^{4 / 3} .
\end{aligned}
$$

The corresponding proton isotropisation time scale is

$t_{\mathrm{t}, \mathrm{p}}=\frac{\lambda_{\mathrm{p}}}{c}=\frac{3 \sqrt{2}}{8 \pi} \frac{1}{\omega_{\mathrm{p}, \mathrm{e}}} \frac{n_{b}}{n_{i}}\left(\frac{m_{\mathrm{p}} \Gamma}{m_{\mathrm{e}}}\right)^{4 / 3}$.

Using the proton resonance approximation (103) we obtain the smallest resonant wavenumbers at $\mu= \pm 1$ from the condition $g_{\mathrm{p}}(\mu= \pm 1, x)=0$ as

$x_{\mathrm{p}, \min } \simeq \mp \frac{\epsilon}{\gamma_{\mathrm{p}}}$

which corresponds to

$\left|k_{\mathrm{p}, \min }\right|=k_{\mathrm{e}}\left|x_{\mathrm{p}, \min }\right|=\frac{m_{\mathrm{e}}}{m_{\mathrm{p}} \Gamma} \frac{\Omega_{\mathrm{e}}}{v}=\frac{1}{R_{\mathrm{p}}}$.

This indicates that the beam protons resonate with Alfven waves with wavenumbers larger than the inverse of the proton gyroradius.

\subsubsection{Scattering length of beam electrons}

The function $g_{i}(\mu, x)$ appearing in the Fokker-Planck coefficient (89) for beam electrons is

$g_{\mathrm{e}}(\mu, x)=\frac{\mu}{\epsilon}+\left(1+x^{2}\right)^{-1 / 2}-\left(\Gamma_{\mathrm{e}} x\right)^{-1}$

which we approximate as

$g_{\mathrm{e}}(\mu, x) \simeq \begin{cases}\frac{\mu}{\epsilon}-\frac{1}{x \Gamma_{\mathrm{e}}} & \text { for }-1 \leq \mu \leq-\epsilon \\ \frac{\mu}{\epsilon}+\frac{\Gamma_{\mathrm{e}} x-1}{\Gamma_{\mathrm{e}}^{2 / 3}-1} & \text { for }-\epsilon \leq \mu \leq 0 \\ \frac{\mu}{\epsilon}+1-\frac{1}{\Gamma_{\mathrm{e}} x} & \text { for } 0 \leq \mu \leq 1 .\end{cases}$

The electron Fokker-Planck coefficient then is approximated as $D_{\mu \mu}^{\mathrm{e}}(\mu) \simeq \frac{d_{\mathrm{e}} \Gamma_{\mathrm{e}}^{2}\left(1-\mu^{2}\right)}{V_{\mathrm{e}} k_{\mathrm{e}}^{2}}$

$\begin{cases}\frac{|\mu|}{\epsilon} & \text { for }-1 \leq \mu \leq-\epsilon \\ \left(\Gamma_{\mathrm{e}}^{2 / 3}-1\right)\left|\left(\Gamma_{\mathrm{e}}^{2 / 3}-1\right) \frac{\mu}{\epsilon}-1\right|^{-3} & \text { for }-\epsilon \leq \mu \leq 0 \\ \left|\frac{\mu}{\epsilon}+1\right| & \text { for } 0 \leq \mu \leq 1 .\end{cases}$

Inserting Eq. (111) into Eq. (92) gives the electron scattering length

$\lambda_{\mathrm{e}} \simeq \frac{3}{32} \frac{V_{\mathrm{e}}^{2} k_{\mathrm{e}}^{2}}{d_{\mathrm{e}} \Gamma_{\mathrm{e}}^{2 / 3}}=\frac{3 \sqrt{2}}{8 \pi} \frac{c}{\omega_{\mathrm{p}, \mathrm{e}}} \frac{n_{b}}{n_{i}} \Gamma^{4 / 3}$

and the corresponding electron isotropisation time scale

$t_{\mathrm{t}, \mathrm{e}}=\frac{\lambda_{\mathrm{e}}}{c}=\frac{3 \sqrt{2}}{8 \pi} \frac{1}{\omega_{\mathrm{p}, \mathrm{e}}} \frac{n_{b}}{n_{i}} \Gamma^{4 / 3}$.

Using the electron resonance approximation (110) we obtain the smallest resonant wavenumbers at $\mu= \pm 1$ from the condition $g_{\mathrm{e}}(\mu= \pm 1, x)=0$ as

$x_{\mathrm{e}, \min } \simeq \pm \frac{\epsilon}{\Gamma_{\mathrm{e}}}$

which corresponds to

$\left|k_{\mathrm{e}, \min }\right|=k_{\mathrm{e}}\left|x_{\mathrm{e}, \min }\right|=\frac{\Omega_{\mathrm{e}}}{v \Gamma}=\frac{1}{R_{\mathrm{e}}}$.

The beam electrons resonate with Alfven waves with wavenumbers larger than the inverse of the electron gyroradius. Therefore, isotropisation of the beam electrons is possible with their own self-generated turbulence, even in cases where the beam protons would not generate transverse turbulence. 


\subsection{Electrostatic or transverse beam relaxation: which is faster?}

By comparing the respective time scales, Eqs. (72), (71), (113) and (106), we can answer the important question which beam relaxation process in pair plasmas is faster; plateauing in parallel momentum by the electrostatic instability or pitch-angle isotropisation by the transverse instability.

For the beam electrons we obtain the ratio of relaxation times

$\frac{t_{\mathrm{e}, \mathrm{e}}}{t_{\mathrm{t}, \mathrm{e}}}=\frac{8}{3} \frac{m_{\mathrm{e}}}{m_{\mathrm{p}}} \Gamma^{-7 / 3} \ll 1$

while for the beam protons

$\frac{t_{\mathrm{e}, \mathrm{p}}}{t_{\mathrm{t}, \mathrm{p}}}=\frac{8}{3}\left(\frac{m_{\mathrm{e}}}{m_{\mathrm{p}}}\right)^{1 / 3} \Gamma^{-7 / 3} \ll 1$.

In both cases the electrostatic relaxation time is much smaller than the transverse relaxation time, so that the incoming interstellar protons and electrons first relax to the plateau distribution (63) in parallel momentum on the time sales $t_{\mathrm{e}, \mathrm{e}}$ and $t_{\mathrm{e}, \mathrm{p}}$. As a consequence, the initial energy density of the beam particles reduces to half of its value; the other half has been transformed to electric field fluctuations of the excited electrostatic turbulence (see Eq. (69)).

We can now study the secular evolution after time $t_{\mathrm{e}, \mathrm{e}}$ and $t_{\mathrm{e}, \mathrm{p}}$, respectively, of the plateau distribution (63) which is still unstable with respect to the excitation of transverse waves.

\section{Secular transverse beam relaxation}

We again use the derived quasilinear integrals for transverse beam evolution during the secular phase. Here the boundary conditions are modified: at the beginning of the secular phase $\left(t=t_{\mathrm{e}}\right)$ the interstellar electrons and protons obey the plateau distribution

$G_{i}\left(p_{\|}, t_{\mathrm{e}}\right)=\frac{1}{P} H\left[p_{\|}+P\right] H\left[-p_{\|}\right]$

corresponding to

$f_{i}\left(p, \mu, t_{\mathrm{e}}\right)=\frac{1}{2 \pi P p^{2}} \delta(\mu+1) H[P-p]$.

Again the final state of the evolution of the transverse instability is reached at time $t_{\mathrm{s}}$ when both growth rate and temporal derivative of the distribution are zero, i.e. when $\partial F_{i} / \partial \mu=0$, corresponding to

$f_{i}\left(p, \mu, t=t_{\mathrm{s}}\right)=\frac{H[P-p]}{4 \pi P p^{2}}$

At this time the magnetohydrodynamic waves have completely isotropised the plateau distribution.
Starting from Eqs. (31) and (40) we can repeat the earlier analysis mutatis mutandi to derive instead of Eq. (75) that in this case

$Z(k)=\frac{2 \pi V_{\mathrm{e}} B^{2}}{|k| c^{2}} \sum_{i} \frac{\omega_{\mathrm{p}, i}^{2}}{\Omega_{i}^{2}} \int_{m_{i}\left|x_{i}\right|}^{\infty} \mathrm{d} p p^{2} v \sqrt{1+\frac{p^{2}}{m_{i}^{2} c^{2}}}\left|\mu_{\mathrm{c}}\right|$

$\int_{-1}^{\mu_{\mathrm{c}}} \mathrm{d} \mu\left[f_{i}\left(p, \mu, t_{\mathrm{s}}\right)-f_{i}\left(p, \mu, t_{\mathrm{e}}\right)\right]=\frac{2 \pi V_{\mathrm{e}} B^{2}}{k^{2} c^{2}} \sum_{i} \frac{\omega_{\mathrm{p}, i}^{2}}{\left|\Omega_{i}\right|}$

$\int_{m_{i}\left|\Omega_{i} / k\right|}^{\infty} \mathrm{d} p p^{2} \int_{-1}^{\mu_{\mathrm{c}}} \mathrm{d} \mu\left[f_{i}\left(p, \mu, t_{\mathrm{s}}\right)-f_{i}\left(p, \mu, t_{\mathrm{e}}\right)\right]$.

Using Eqs. (119)-(120) we obtain

$\int_{-1}^{\mu_{\mathrm{c}}} \mathrm{d} \mu\left[f_{i}\left(p, \mu, t_{\mathrm{s}}\right)-f_{i}\left(p, \mu, t_{\mathrm{e}}\right)\right]=-\frac{1-\mu_{\mathrm{c}}}{4 \pi p^{2} P} H[P-p]$

which after momentum integration yields for Eq. (121)

$Z(k)=-\frac{V_{\mathrm{e}} B^{2}}{2 k^{2} c^{2}} \sum_{i} \frac{\omega_{\mathrm{p}, i}^{2}}{\left|\Omega_{i}\right|}\left[1-\frac{1}{|k| R_{i}}+\frac{\operatorname{sgn}\left(q_{i}\right)}{k R_{i}} \ln \left(|k| R_{i}\right)\right]$

$H\left[|k|-R_{i}^{-1}\right]=-\frac{V_{\mathrm{e}} B^{2}}{2 k^{2} c^{2} V \Gamma} \sum_{i} \omega_{\mathrm{p}, i}^{2} R_{i}$

$\left[1-\frac{1}{|k| R_{i}}+\frac{\operatorname{sgn}\left(q_{i}\right)}{k R_{i}} \ln \left(|k| R_{i}\right)\right] H\left[|k|-R_{i}^{-1}\right]$

which also results from applying the bi-spherical shell method of Appendix C to the flat distribution (119). For large wavenumbers $|k| R_{\mathrm{p}}>>1$ Eq. (123) is well approximated by

$Z(k) \simeq-\frac{V_{\mathrm{e}} B^{2}}{2 k^{2} c^{2} V \Gamma} \sum_{i} \omega_{\mathrm{p}, i}^{2} R_{i}$

which is identical to the previous result (75). Again $Z(k)$ is negative for all wavenumber values, implying according to Eq. (61) that predominantly backward moving left-handed and right-handed polarized Alfven waves are generated. If the transverse turbulence at time $t_{\mathrm{e}}$ is negligibly small the respective intensities of the fully developed turbulence at the end of the secular evolution phase are

$I_{-}\left(k, t_{\mathrm{s}}\right)=|Z(|k|)|, \quad I_{+}\left(k, t_{\mathrm{s}}\right)=0$.

The generated wavenumber spectrum and the total change in transverse wave energy density is independent whether one starts from a monoenergetic beam (as we did in the initial phase of the evolution) or from a plateau distribution resulting from the faster electrostatic relaxation (as we did in the secular phase). This implies that we get exactly the same time scales for the pitch-angle isotropisation, (106) and (113), of the plateau distribution function, so that at the end of the secular phase, $t_{\mathrm{s}}=t_{\mathrm{t}, \mathrm{e}}$ for electrons and $t_{\mathrm{s}}=t_{\mathrm{t}, \mathrm{p}}$ for protons, the sweptup interstellar protons and electrons have a flat and isotropic differential momentum spectrum

$N_{i}\left(p, t_{\mathrm{s}}\right)=4 \pi p^{2} f_{i}\left(p, \mu, t=t_{\mathrm{s}}\right)=\frac{H[P-p]}{P}$

in the rest frame of the plasma blob. 


\section{Application to AGN jet outflows}

In order for the electrostatic and the transverse instability to plateau and isotropise the incoming interstellar electron and proton beam distributions, the respective co-moving time scales $t_{\mathrm{e}}$ and $t_{\mathrm{t}}$ have to be smaller than the crossing time of the beam particles which is given by the co-moving light crossing time of the blob

$t_{1}=d / c=3 \times 10^{4} d_{13}^{*} \Gamma_{2} \mathrm{~s}$.

We consider these conditions separately for beam plateauing and beam isotropisation.

\subsection{Beam plateauing}

According to Eqs. (72) and (127) for beam electrons the requirement $t_{\mathrm{e}, \mathrm{e}}<t_{1}$ leads to

$\frac{\sqrt{2}}{\pi \omega_{\mathrm{p}, \mathrm{e}}} \frac{m_{\mathrm{e}}}{m_{\mathrm{p}}} \frac{n_{b}^{*}}{n_{i}^{*} \Gamma^{3}}<3 \times 10^{4} d_{13}^{*} \Gamma_{2}$,

which is identical to

$\frac{\left(n_{b, 10}^{*}\right)^{1 / 2}}{n_{i}^{*} d_{13}^{*} \Gamma_{2}^{7 / 2}}<6.9 \times 10^{12}$,

which is well fulfilled for standard AGN jet outflow and environment parameters.

Likewise with Eq. (73) for beam protons the requirement $t_{\mathrm{e}, \mathrm{p}}<t_{1}$ becomes

$\frac{\left(n_{b, 10}^{*}\right)^{1 / 2}}{n_{i}^{*} d_{13}^{*} \Gamma_{2}^{7 / 2}}<2.0 \times 10^{6}$

which is also fulfilled for standard jet outflow and environment parameters.

We therefore conclude that for standard AGN jet outflow and environment parameters the initial beam distributions of interstellar protons and electrons relax to plataeu distributions in parallel momentum, transferring thereby one-half of the initial energy density of the beam particles to electric field fluctuations of the generated electrostatic turbulence.

\subsection{Beam isotropisation and generation of radiation}

According to Eqs. (113) and (127) for plateaued electrons the requirement $t_{\mathrm{t}, \mathrm{e}}<t_{1}$ leads to

$$
\frac{\left(n_{b, 10}^{*}\right)^{1 / 2}}{n_{i}^{*} d_{13}^{*} \Gamma_{2}^{7 / 6}}<10^{8}
$$

which is fulfilled for standard jet outflow and environment parameters.

For plateaued protons Eqs. (106) and (127) imply

$$
\frac{\left(n_{b, 10}^{*}\right)^{1 / 2}}{n_{i}^{*} d_{13}^{*} \Gamma_{2}^{7 / 6}}<4.4 \times 10^{3}
$$

which is also fulfilled for the adopted standard jet outflow and environment parameters.
If we take the adopted AGN jet outflow $\left(n_{b, 10}^{*}=1, \Gamma_{2}=1\right.$, $\left.d_{13}^{*}=1\right)$ and environment $\left(n_{i}^{*}\right)$ parameters as face-value parameters, we conclude that the interstellar electrons and protons will isotropise by their self-generated transverse turbulence and thus be picked-up by the outflow pair plasma. In Appendix D we demonstrate that both transverse wave isotropisation time scales (113) and (106) are much shorter than the isotropisation of beam electrons and protons by elastic Coulomb interactions with pair jet plasma.

In the pair outflow frame the external density is $n_{i}=\Gamma n_{i}^{*}$ and pick-up of interstellar protons and electrons occurs at a rate

$\dot{N}_{i}(\gamma)=\pi r^{2} c n_{i}^{*} \sqrt{\Gamma^{2}-1} \delta(\gamma-\Gamma)$.

The pick-up is a source of isotropic, quasi-monoergetic protons and electrons with Lorentz factor $\Gamma$ in the jet outflow frame. By interacting with the pair background plasma (electron bremsstrahlung, proton inverse bremsstrahlung), the ordered magnetic field (synchrotron radiation) and internal and external target photon field (inverse Compton scattering) the pick-up electrons and protons generate nonthermal photons. The detailed modelling of the radiation signature of this leptonic jet outflow, including the time evolution of the energy spectra of radiating particles and the transformation to the observer's frame, is analogous to the earlier treatment of the hadronic jet outflow (see Pohl \& Schlickeiser 2000), but lies beyond the scope of the present paper. We note, however, one important difference: the pick-up protons do not find thermal protons in the background pair plasma to undergo inelastic $p-p$-collisions, so that no secondary neutral pions, secondary electrons and positrons and neutrinos will be produced. These radiation products, that provided the dominating contribution of the Doppler-boosted GeV-TeV photon emission in the hadronic jet model and gave rise to correlated Dopplerboosted GeV-TeV neutrino emission (Schuster et al. 2002), will be absent here. The pick-up protons, that carry a factor $m_{\mathrm{p}} / m_{\mathrm{e}}=1836$ more power than the pick-up electrons, can only radiate by the much less efficient inverse bremsstrahlung process. High-energy GeV-TeV emission in the leptonic jet outflow model will be dominated by inverse Compton scattering of pick-up electrons off internal and external target photon fields. It will be most interesting to study in future work quantitatively the leptonic jet radiation signatures modifying the formalism of Böttcher et al. (2001) developed for optically-thick collisiondominated relativistic pair gamma-ray burst fireballs.

\subsection{Build-up of transverse magnetohydrodynamic turbulence}

The co-moving time scales

$t_{\mathrm{t}, \mathrm{e}}=0.19 \frac{\left(n_{b, 10}^{*}\right)^{1 / 2}}{n_{i}^{*} \Gamma_{2}^{1 / 6}} \mathrm{~s}$

and

$t_{\mathrm{t}, \mathrm{p}}=\left(\frac{m_{\mathrm{p}}}{m_{\mathrm{e}}}\right)^{4 / 3} t_{\mathrm{t}, \mathrm{e}}=4.3 \times 10^{3} \frac{\left(n_{b, 10}^{*}\right)^{1 / 2}}{n_{i}^{*} \Gamma_{2}^{1 / 6}} \mathrm{~s}$ 
given in Eqs. (113) and (106), respectively, also provide estimates for the time to build up the fully developed power spectrum of transverse hydromagnetic turbulence from the beam electrons and the beam protons, respectively. Hydromagnetic turbulence upstream and downstream is crucial for diffusive shock acceleration to operate. We recall that beam electrons alone only produce transverse turbulence at wavenumbers larger than the inverse of the electron gyroradius $(|k| \geq$ $R_{\mathrm{e}}^{-1}$ ) with an (integrated) energy density smaller by a factor $\left(m_{\mathrm{e}} / m_{\mathrm{p}}\right)=1 / 1836$ than the turbulence produced by the beam protons. As our earlier estimate (130) indicated, this small fraction of electron generated transverse turbulence is built up after time $t_{\mathrm{t}, \mathrm{e}}$ for standard AGN outflow and environment parameters. For the protons to provide the much larger fraction of transverse turbulence, the longer time $t_{\mathrm{t}, \mathrm{p}}$ is needed, and it will occur at later times of the AGN jet evolution. The co-moving time scales (133)-(134) correspond to Dopplershortened time scales $t_{\mathrm{t}}^{*}=t_{\mathrm{t}} / D_{\mathrm{L}}$, where the Doppler factor $D_{\mathrm{L}}=\Gamma^{-1}\left[1-\left(V \cos \theta^{*} / c\right)\right]^{-1}$, depends on the observer's viewing angle $\theta^{*}$.

Equations (133) and (134) indicate that it takes a nonnegligible fraction of the early AGN jet phase, to build up the magnetohydrodynamic turbulence for nonthermal particle acceleration at either internal or external shocks associated with the pair jet outflow.

\section{Summary and conclusions}

We have investigated the microphysical details of the energy conversion in relativistic pair outflows interacting with the surrounding interstellar medium consisting of cold protons and electrons. We have represented the relativistic pair blast wave as a one-dimensional channeled outflow directed parallel to a uniform background magnetic field. Viewed from the coordinate system comoving with the pair outflow, the interstellar protons and electrons represent a proton-electron beam propagating with relativistic speed antiparallel to the uniform magnetic field direction. We demonstrate that the beam excites both electrostatic and low-frequency magnetohydrodynamic Alfven-type waves via a two-stream instability in the pair background plasma, and we calculate the time evolution of the distribution functions of the beam particles and the generated plasma wave turbulence power spectra.

For standard AGN jet outflow and environment parameters we show that the initial beam distributions of interstellar protons and electrons quickly relax to plateau-distributions in parallel momentum, transferring thereby one-half of the initial energy density of the beam particles to electric field fluctuations of the generated electrostatic turbulence.

On considerably longer time scales, the plateaued interstellar electrons and protons will isotropise by their self-generated transverse turbulence and thus be picked-up in the outflow pair plasma. These longer time scale are also characteristic for establishing fully developed power spectra of transverse hydromagnetic turbulence from the plateaued electrons and protons. This hydromagnetic turbulence upstream and downstream is crucial for diffusive shock acceleration to operate at external or internal shocks associated with pair outflows. It takes a finite time period of the early AGN outflow phase to build up the magnetohydrodynamic turbulence for nonthermal particle acceleration at either internal or external shocks associated with pair jet outflows. During this initial phase - prior to the generation of hydromagnetic turbulence - the radiative and energyexchange processes in the jet outflow are not dominated by acceleration processes of nonthermal charged particles at collisionless shock waves.

Acknowledgements. We gratefully acknowledge the helpful and constructive comments of the referee. RS and IL acknowledge support by the Deutsche Forschungsgemeinschaft through Sonderforschungsbereich 191. RV acknowledges the financial support of the Academy of Finland (project 46331) and the PLATON network (EC contract HPRN-CT-2000-00153). The work of MB is supported by NASA through Chandra Postdoctoral Fellowship grant PF 9-10007 awarded by the Chandra X-ray Center, which is operated by the Smithsonian Astrophysical Observatory for NASA under contract NAS 8-39073. RS, CS and MP acknowledge support by the Verbundforschung, grants DESY AG9PCA7 and DESY CH1PCA6.

\section{Appendix A: Dispersion relations of parallel plasma waves in a thermal magnetised pair plasma}

The properties of parallel propagating plasma waves in magnetised thermal plasmas have been discussed by Fichtner \& Schlickeiser (1995) and Schlickeiser et al. (1997). For subluminal waves with index of refraction $N=c k / \omega_{\mathrm{R}}>1$ the dispersion relations of longitudinal $\left(\Lambda_{\mathrm{L}}=0\right)$ and transverse $\left(\Lambda_{\mathrm{T}}=0\right)$ can be split in their real and imaginary parts.

\subsection{Longitudinal waves}

According to Eq. (22) of Fichtner \& Schlickeiser (1995) we obtain for longitudinal waves in the limit of weak damping $\omega=$ $\omega_{\mathrm{R}}+\imath \omega_{\mathrm{I}}, \omega_{\mathrm{I}}<<\omega_{\mathrm{R}}$

$$
\begin{aligned}
& \mathfrak{R} \Lambda_{\mathrm{L}}\left(\omega_{\mathrm{R}}, k\right)=1+\frac{\sigma^{2}\left(1+2 \sigma^{2}\right)}{\omega_{\mathrm{R}}^{2}} \sum_{a} \omega_{\mathrm{p}, a}^{2} \mu_{a}- \\
& -\sum_{a} \frac{\omega_{\mathrm{p}, a}^{2} \mu_{a}^{2} \sigma^{3}}{\omega_{\mathrm{R}}^{2} K_{2}\left(\mu_{a}\right)} \int_{0}^{\sigma} \mathrm{d} y\left(1+\sigma^{2}+y^{2}\right) \frac{K_{3}\left(\mu_{a} \sqrt{1+\sigma^{2}-y^{2}}\right.}{\left(1+\sigma^{2}-y^{2}\right)^{3 / 2}}=0
\end{aligned}
$$

and

$$
\begin{aligned}
& \mathfrak{J} \Lambda_{\mathrm{L}}\left(\omega_{\mathrm{R}}, k\right)=\frac{\pi}{2} \operatorname{sgn}\left(\omega_{\mathrm{R}}\right) \sum_{\mathrm{a}} \frac{\omega_{\mathrm{p}, a}^{2} \exp \left(-\mu_{\mathrm{a}} \sqrt{1+\sigma^{2}}\right)}{\omega_{\mathrm{R}}^{2} N^{3} \mu_{a} K_{2}\left(\mu_{a}\right)} \\
& {\left[2+2 \mu_{a} \sqrt{1+\sigma^{2}}+\mu_{a}^{2}\left(1+\sigma^{2}\right)\right]=0}
\end{aligned}
$$

where $\sigma=\left(N^{2}-1\right)^{-1 / 2}, \mu_{a}=1 / \Theta_{a}=m_{a} c^{2} /\left(k_{\mathrm{B}} T_{a}\right)$, and $\omega_{\mathrm{p}, a}$ denotes the plasma frequency of species $a$. Solutions of Eq. (135) define the frequency-wavenumber relations $\omega_{\mathrm{R}}=\omega_{\mathrm{R}}(k)$ of all possible modes in the system whereas the growth or damping rate of each mode is calculated from Eqs. (135) and (136) by the standard weak-damping expansion

$\omega_{\mathrm{I}}=-\frac{\mathfrak{J} \Lambda_{\mathrm{L}}\left(\omega_{\mathrm{R}}, k\right)}{\left[\frac{\partial \mathfrak{R} \Lambda_{\mathrm{L}}\left(\omega_{\mathrm{R}}, k\right)}{\partial \omega_{\mathrm{R}}}\right]}$ 
The integral equation ((135) has been further reduced by Schlickeiser \& Mause (1997). Introducing normalised wavenumbers $\kappa=c k / \omega_{\mathrm{p}, \mathrm{e}}$ and frequencies $f=\omega_{\mathrm{R}} / \omega_{\mathrm{p}, \mathrm{e}}$ these authors obtained for non-relativistic temperatures $\mu_{a}>>$ 1 (see Eqs. (34)-(38) of Schlickeiser \& Mause 1997) the approximation

$\mathfrak{R} \Lambda_{\mathrm{L}}(f, \kappa) \simeq 1-\sum_{a} \frac{\xi_{a}}{f^{2}}\left[1-\frac{5}{2 \mu_{a}}+\frac{3 \kappa^{2}}{\mu_{a} f^{2}}\right]=0$

with $\xi_{a}=\omega_{\mathrm{p}, a} / \omega_{\mathrm{p}, \mathrm{e}}$. For a single temperature plasma this approximation holds for the wavenumber range

$\left[\sum_{a}\left(1+\frac{1}{2 \mu_{a}}\right)\right]^{1 / 2} \leq|\kappa| \leq 2 \mu_{a}^{1 / 2}\left[1-\frac{5}{16 \mu_{a}}\right]$.

For a single-temperature $\left(\mu_{a}=1 / \Theta\right)$ non-relativistic electronpositron plasma to lowest order in the normalised temperature $\Theta<<1$, Eq. (138) reduces to

$\mathfrak{R} \Lambda_{\mathrm{L}}\left(\omega_{\mathrm{R}}, k\right) \simeq 1-\frac{2 \omega_{\mathrm{p}, \mathrm{e}}^{2}}{\omega_{\mathrm{R}}^{2}}=0$

yielding electrostatic waves

$\omega_{\mathrm{R}}^{2}=2 \omega_{\mathrm{p}, \mathrm{e}}^{2}$

in the wavenumber range $|k| \leq 2 \omega_{\mathrm{p}, \mathrm{e}} /\left(c \Theta^{1 / 2}\right)$ as only longitudinal plasma mode. Moreover, we obtain

$\frac{\partial \Re \Lambda_{\mathrm{L}}\left(\omega_{\mathrm{R}}, k\right)}{\partial \omega_{\mathrm{R}}}=\frac{4 \omega_{\mathrm{p}, \mathrm{e}}^{2}}{\omega_{\mathrm{R}}^{3}}=\frac{2}{\omega_{\mathrm{R}}}$.

While superluminal electrostatic waves are not damped, in the subluminal wavenumber range $\sqrt{2} \omega_{\mathrm{p}, \mathrm{e}} / c \leq|k| \leq 2 \omega_{\mathrm{p}, \mathrm{e}} /\left(c \Theta^{1 / 2}\right)$ we derive for the associated damping rate (136)

$$
\begin{aligned}
& \omega_{\mathrm{I}}=-\frac{\omega_{\mathrm{R}}}{2} \mathfrak{J} \Lambda_{\mathrm{L}}\left(\omega_{\mathrm{R}}, k\right)=-\pi \operatorname{sgn}\left(\omega_{\mathrm{R}}\right) \frac{\omega_{\mathrm{p}, \mathrm{e}}^{4} \Theta}{c^{3} k^{3} K_{2}(1 / \Theta)} \\
& \exp \left(-\sqrt{1+\sigma^{2}} / \Theta\right)\left[2+\frac{2 \sqrt{1+\sigma^{2}}}{\Theta}+\frac{\left(1+\sigma^{2}\right)}{\Theta^{2}}\right] \\
& =-\pi \operatorname{sgn}\left(\omega_{\mathrm{R}}\right) \frac{\omega_{\mathrm{p}, \mathrm{e}}^{4}}{c k\left[c^{2} k^{2}-2 \omega_{\mathrm{p}, \mathrm{e}}^{2}\right] \Theta K_{2}(1 / \Theta)} \\
& \exp \left[-\frac{c|k|}{\Theta \sqrt{c^{2} k^{2}-2 \omega_{\mathrm{p}, \mathrm{e}}^{2}}}\right] \\
& {\left[1+\frac{2 \Theta}{c|k|} \sqrt{c^{2} k^{2}-2 \omega_{\mathrm{p}, \mathrm{e}}^{2}}+\frac{2 \Theta^{2}\left(c^{2} k^{2}-2 \omega_{\mathrm{p}, \mathrm{e}}^{2}\right)}{c^{2} k^{2}}\right] .}
\end{aligned}
$$

\subsection{Transverse waves}

The dispersion relation of parallel propagating transverse waves in a thermal plasma with non-relativistic temperatures has been analysed in Sect. 3 of Schlickeiser et al. (1997). Its real part

$\mathfrak{R} \Lambda_{\mathrm{T}}=1-N^{2}-\sum_{a} \frac{\omega_{\mathrm{p}, a}^{2}}{k v_{\mathrm{th}, a} \omega_{\mathrm{R}}} Z\left(\frac{\omega_{\mathrm{R}}-\Omega_{a}}{k v_{\mathrm{th}, a}}\right)=0$ involves the plasma dispersion function $Z(x)$ (Fried \& Conte $1962)$, the non-relativistic gyrofrequencies $\Omega_{a}=q_{a} B /\left(m_{a} c\right)$ and the non-relativistic thermal velocities $v_{\mathrm{th}, a}^{2}=2 c^{2} / \mu_{a}=$ $2 k_{\mathrm{B}} T_{a} / m_{a}$. In Eq. (144) we adopt the convention that positive values of $\omega_{\mathrm{R}}>0$ denote left-handed circularly polarized waves, and that negative values of $\omega_{\mathrm{R}}<0$ denote right-handed circularly polarized waves. An expansion of $Z(x)$ for large arguments and the weak-damping approximation $\left|\omega_{\mathrm{I}} / \omega_{\mathrm{R}}\right| \ll 1$ allows the derivation of a simplified, asymptotic dispersion relation for transverse oscillations propagating parallel to the external magnetic field

$\mathfrak{R} \Lambda_{\mathrm{T}} \simeq 1-\frac{c^{2} k^{2}}{\omega_{\mathrm{R}}^{2}}-\sum_{a} \frac{\omega_{\mathrm{p}, a}^{2}}{\omega_{\mathrm{R}}\left(\omega_{\mathrm{R}}-\Omega_{a}\right)}=0$.

According to Eq. (54) of Schlickeiser et al. (1997) we obtain for the weak damping rate in the same limit

$\omega_{\mathrm{I}}=-\frac{\pi^{1 / 2}}{\omega_{\mathrm{R}}} \operatorname{sgn}\left(\omega_{\mathrm{R}}\right)\left[\frac{\partial \mathfrak{R} \Lambda_{\mathrm{T}}\left(\omega_{\mathrm{R}}, k\right)}{\partial \omega_{\mathrm{R}}}\right]^{-1}$

$\sum_{a} \frac{\omega_{\mathrm{p}, a}^{2}}{k v_{\mathrm{th}, a}} \exp \left(-\left[\frac{\omega_{\mathrm{R}}-\Omega_{a}}{k v_{\mathrm{th}, a}}\right]^{2}\right)$.

For a single-temperature $\left(\mu_{a}=1 / \Theta\right)$ non-relativistic electronpositron plasma Eq. (145) reduces to

$\mathfrak{R} \Lambda_{\mathrm{T}} \simeq 1-\frac{c^{2} k^{2}}{\omega_{\mathrm{R}}^{2}}-\frac{2 \omega_{\mathrm{p}, \mathrm{e}}^{2}}{\omega_{\mathrm{R}}^{2}-\Omega_{\mathrm{e}}^{2}}=0$.

For subluminal phase speeds $\left(\left|\omega_{\mathrm{R}} / k\right|<<c\right)$ we may neglect the first term on the right hand side of Eq. (147), so that the transverse dispersion relation yields

$\omega_{\mathrm{R}}^{2} \simeq \frac{V_{\mathrm{e}}^{2} \Omega_{\mathrm{e}}^{2} k^{2}}{\Omega_{\mathrm{e}}^{2}+V_{\mathrm{e}}^{2} k^{2}}$

where

$V_{\mathrm{e}}=\frac{\Omega_{\mathrm{e}} c}{\sqrt{2} \omega_{\mathrm{p}, \mathrm{e}}}=B\left[8 \pi n_{b} m_{\mathrm{e}}\right]^{-1 / 2}=$

$6.6 \times 10^{12}(B /$ Gauss $)\left(n_{\mathrm{b}} / \mathrm{cm}^{-3}\right)^{-1 / 2} \mathrm{~cm} \mathrm{~s}^{-1}=$

$9.2 \times 10^{7}\left(\epsilon_{B,-1} \Theta_{-4}\right)^{1 / 2} \mathrm{~cm} \mathrm{~s}^{-1}$

is the Alfven speed in the pair plasma. For wavenumbers much less than $|k|<<k_{\mathrm{e}}$, where

$k_{\mathrm{e}} \equiv \Omega_{\mathrm{e}} / V_{\mathrm{e}}=2.7 \times 10^{-2} n_{b, 8}^{1 / 2} \mathrm{~cm}^{-1}$

is the inverse pair skin length, Eq. (148) reduces to

$\omega_{\mathrm{R}}^{2}=V_{\mathrm{e}}^{2} k^{2}$

whereas in the opposite case $|k|>>k_{\mathrm{e}}$ Eq. (148) approaches the pair-cyclotron wave limit $\omega_{\mathrm{R}}^{2} \simeq \Omega_{\mathrm{e}}^{2}$.

Moreover, Eq. (147) yields

$\frac{\partial \mathfrak{R} \Lambda_{\mathrm{T}}\left(\omega_{\mathrm{R}}, k\right)}{\partial \omega_{\mathrm{R}}}=\frac{4 \omega_{\mathrm{p}, \mathrm{e}}^{2} \omega_{\mathrm{R}}}{\left(\omega_{\mathrm{R}}^{2}-\Omega_{\mathrm{e}}^{2}\right)^{2}}+\frac{2 c^{2} k^{2}}{\omega_{\mathrm{R}}^{3}}$ 
so that the damping rate (146) becomes

$$
\begin{aligned}
& \omega_{\mathrm{I}}(k)=-\frac{1}{2} \sqrt{\frac{\pi}{2 \Theta}} \frac{\omega_{\mathrm{p}, \mathrm{e}}^{2}}{c k} \frac{\operatorname{sgn}\left(\omega_{\mathrm{R}}\right)}{\left[\frac{c^{2} k^{2}}{\omega_{\mathrm{R}}^{2}}+\frac{2 \omega_{\mathrm{p}, \mathrm{e}}^{2} \omega_{\mathrm{R}}^{2}}{\left(\omega_{\mathrm{R}}^{2}-\Omega_{\mathrm{e}}^{2}\right)^{2}}\right]} \\
& {\left[\exp \left(-\frac{\left(\omega_{\mathrm{R}}-\Omega_{\mathrm{e}}\right)^{2}}{2 \Theta k^{2} c^{2}}\right)+\exp \left(-\frac{\left(\omega_{\mathrm{R}}+\Omega_{\mathrm{e}}\right)^{2}}{2 \Theta k^{2} c^{2}}\right)\right] .}
\end{aligned}
$$

Especially, for small frequencies $\left(\left|\omega_{R}\right|<<\Omega_{\mathrm{e}}\right)$ we obtain for the pair Alfven waves (151) the damping rate

$\omega_{\mathrm{I}, \mathrm{A}}(k)=-\frac{\pi^{1 / 2}}{2} \frac{\Omega_{\mathrm{e}}^{2}}{v_{\text {th,e }}|k|\left(1+\frac{k^{2}}{k_{\mathrm{e}}^{2}}\right)} \exp \left(-\left(\frac{\Omega_{\mathrm{e}}}{v_{\text {th,e }} k}\right)^{2}\right)$

$\simeq-\frac{\pi^{1 / 2}}{2} \frac{\Omega_{\mathrm{e}} V_{\mathrm{e}}}{v_{\text {th,e }}} \frac{k_{\mathrm{e}}}{|k|} \exp \left(-\left(\frac{V_{\mathrm{e}} k_{\mathrm{e}}}{v_{\text {th, }} k}\right)^{2}\right)$

where $v_{\text {th,e }}=\sqrt{2 \Theta} c=\sqrt{2 k_{\mathrm{B}} T_{\mathrm{e}} / m_{\mathrm{e}}}$ is the non-relativistic thermal pair velocity.

To check the weak-damping approximation we calculate

$\left|\omega_{\mathrm{R}} / \omega_{I}\right|=\frac{\pi^{1 / 2}}{2} \beta_{\mathrm{p}}^{1 / 2} \frac{k_{\mathrm{e}}^{2}}{k^{2}} \exp \left[-\frac{\beta_{\mathrm{p}} k_{\mathrm{e}}^{2}}{k^{2}}\right]$

where we introduce the pair plasma beta $\beta_{\mathrm{p}}=V_{\mathrm{e}}^{2} / v_{\text {th.e }}^{2}=$ $0.05 \epsilon_{\mathrm{B},-1}$. One notices that for small wavenumber values $|k|<<$ $\beta_{\mathrm{p}}^{1 / 2} k_{\mathrm{e}}=0.22 \epsilon_{\mathrm{B},-1}^{1 / 2} k_{\mathrm{e}}$ the weak-damping approximation is well satisfied.

\section{Appendix B: Comparison of the initial growth rates with the thermal Landau and cyclotron damping rates}

We noted that plasma waves, due to the unstable interstellar beam particles entering the jet outflow pair plasma will only grow if the initial growth rate is larger than the thermal Landau (143) and cyclotron (154) damping rates. Here we investigate under which conditions this is the case. We consider longitudinal and transverse waves in turn.

\subsection{Electrostatic waves}

If we include the contributions from the beam particles the initial longitudinal dispersion relation reads

$$
\begin{aligned}
& \Lambda_{\mathrm{e}}(t=0)=\mathfrak{R} \Lambda_{\mathrm{L}}+\imath \mathfrak{J} \Lambda_{\mathrm{L}}+\frac{2 \pi}{\omega} \sum_{i} \omega_{\mathrm{p}, i}^{2} \\
& \times \int_{-\infty}^{\infty} \mathrm{d} p_{\|} \int_{0}^{\infty} \mathrm{d} p_{\perp} \frac{p_{\perp} p_{\|}}{\gamma\left(\omega-k v_{\|}\right)} \frac{\partial f_{i}\left(p_{\perp}, p_{\|}, t=0\right)}{\partial p_{\|}}
\end{aligned}
$$

where $\mathfrak{R} \Lambda_{\mathrm{L}}$ and $\mathfrak{J} \Lambda_{\mathrm{L}}$ are given by Eqs. (140) and (136), respectively. Neglecting $\mathfrak{J} \Lambda_{\mathrm{L}}$ for this calculation we obtain in terms of the normalised phase space distribution (21)

$$
\begin{aligned}
& \Lambda_{\mathrm{e}}(t=0) \simeq \mathfrak{R} \Lambda_{\mathrm{L}}+\frac{1}{\omega} \sum_{i} \omega_{\mathrm{p}, i}^{2} \int_{-\infty}^{\infty} \mathrm{d} p_{\|} p_{\|} \\
& {\left[\left(\sqrt{1+\frac{p_{\|}^{2}}{m_{i}^{2} c^{2}}} \omega-\frac{k p_{\|}}{m_{i}}\right)\right]^{-1} \frac{\partial G_{i}\left(p_{\|}, t=0\right)}{\partial p_{\|}} .}
\end{aligned}
$$

Inserting the initial distribution function (62), i.e. $G\left(p_{\|}, t=\right.$ $0)=\delta\left(p_{\|}+P\right)$, and integrating partially with respect to $p_{\|}$we derive with Eq. (140)

$$
\Lambda_{\mathrm{e}}(t=0)=1-\frac{2 \omega_{\mathrm{p}, \mathrm{e}}^{2}}{\omega^{2}}-\frac{\sum_{i} \omega_{\mathrm{p}, i}^{2}}{\Gamma^{3}(\omega+k V)^{2}}=0 .
$$

With the abbreviations

$w_{\mathrm{e}}=\sqrt{2} \omega_{\mathrm{p}, \mathrm{e}}, \quad w_{i}=\left(\sum_{i} \omega_{\mathrm{p}, i}^{2}\right)^{1 / 2}$

and the substitution

$f=\omega+k V$

Eq. (158) becomes

$|k V-f|=\frac{w_{\mathrm{e}}}{\left(1-\frac{w_{i}^{2}}{\Gamma^{3} f^{2}}\right)^{1 / 2}}$

A posteriori it can be shown that in the resonant wave regime

$w_{i}^{2}<<\Gamma^{3} f^{2}$

so that the sqare root in Eq. (161) can be expanded:

$k V \simeq f-w_{\mathrm{e}}-\frac{w_{\mathrm{e}} w_{i}^{2}}{2 \Gamma^{3} f^{2}}$.

This equation has already been examined by Buneman (1959). Substituting

$f=\frac{w_{\mathrm{e}}^{1 / 3} w_{i}^{2 / 3}}{\Gamma} x, \quad k V=-w_{\mathrm{e}}+\frac{w_{\mathrm{e}}^{1 / 3} w_{i}^{2 / 3} y}{\Gamma}$

Eq. (163) reduces to

$x^{3}-y x^{2}-1 / 2=0$.

This cubic equation has one real and two complex conjugate solutions $x_{1,2}$ provided $y>-3 / 2$, corresponding to wavenumbers

$k>-\left[\frac{3 w_{\mathrm{e}}^{1 / 3} w_{i}^{2 / 3}}{2 \Gamma V}+\frac{w_{\mathrm{e}}}{V}\right]$.

The complex conjugated solutions are

$x_{1,2}=\frac{y}{3}-\frac{1}{2}\left[\left(\frac{1}{4}+\frac{y^{3}}{27}+\Delta^{1 / 2}\right)^{1 / 3}+\left(\frac{1}{4}+\frac{y^{3}}{27}-\Delta^{1 / 2}\right)^{1 / 3}\right]$

$\pm l \frac{\sqrt{3}}{2}\left[\left(\frac{1}{4}+\frac{y^{3}}{27}+\Delta^{1 / 2}\right)^{1 / 3}-\left(\frac{1}{4}+\frac{y^{3}}{27}-\Delta^{1 / 2}\right)^{1 / 3}\right]$

where

$\Delta=\frac{1}{16}+\frac{y^{3}}{54}$

The maximum growth rate $\mathfrak{J} x_{\max }=3^{1 / 2} 2^{-4 / 3}$ occurs for $y=0$, corresponding to

$k_{0}=-w_{\mathrm{e}} / V=-2^{1 / 2} \omega_{\mathrm{p}, \mathrm{e}} / V$. 
Consequently

$\omega_{\mathrm{I}, \max }=\mathfrak{J} \omega_{\max }=\mathfrak{J} f_{\max }=$

$\frac{w_{\mathrm{e}}^{1 / 3} w_{i}^{2 / 3}}{\Gamma} \mathfrak{J} x_{\max }=\frac{3^{1 / 2}}{2 \Gamma} \omega_{\mathrm{p}, \mathrm{e}}^{1 / 3}\left(\sum_{i} \omega_{\mathrm{p}, i}^{2}\right)^{1 / 3}=$

$\frac{3^{1 / 2}}{2} \frac{\omega_{\mathrm{p}, \mathrm{e}}}{\Gamma}\left[\frac{n_{i}}{n_{b}}\left(1+\frac{m_{\mathrm{e}}}{m_{\mathrm{p}}}\right)\right]^{1 / 3}=\frac{3^{1 / 2}}{2} \omega_{\mathrm{p}, \mathrm{e}}\left[\frac{n_{i}^{*}}{\Gamma n_{b}^{*}}\right]^{1 / 3}$.

Now we compare this maximum growth rate with the Landau damping rate (143) calculated at the wavenumber $k_{0}$ which is

$$
\begin{aligned}
& \omega_{I}\left(k_{0}\right)=-\frac{\pi}{2^{3 / 2}} \omega_{\mathrm{p}, \mathrm{e}} \frac{\left(\Gamma^{2}-1\right)^{3 / 2}}{\Gamma} \frac{e^{-\Gamma / \Theta}}{\Theta K_{2}(1 / \Theta)}[1+ \\
& \left.\frac{2 \Theta}{\Gamma}+\frac{2 \Theta^{2}}{\Gamma^{2}}\right] \simeq-\frac{\pi^{1 / 2}}{2 \Theta^{3 / 2}} \omega_{\mathrm{p}, \mathrm{e}} \Gamma^{2} \exp \left[-\frac{\Gamma-1}{\Theta}\right] .
\end{aligned}
$$

For the ratio of the maximum growth rate to this damping rate we obtain

$$
\begin{aligned}
& \frac{\omega_{\mathrm{I}, \max }}{\left|\omega_{i}\left(k_{0}\right)\right|}=\sqrt{3 / \pi}\left(\frac{n_{i}^{*}}{n_{b, 10}^{*}}\right)^{1 / 3} \Theta^{3 / 2} \Gamma^{-7 / 3} \exp \left(+\frac{\Gamma-1}{\Theta}\right) \simeq \\
& \left(n_{i}^{*}\right)^{1 / 3}\left(n_{b, 10}^{*}\right)^{-1 / 3} \Theta_{-4}^{3 / 2} \Gamma_{2}^{-7 / 3} \exp \left[10^{6} \Gamma_{2} \Theta_{-4}^{-1}\right]>>1
\end{aligned}
$$

which is a huge factor for standard AGN jet outflow and environment parameters due to the exponential dependence on $\exp [(\Gamma-1) / \Theta]$. So we can safely neglect the Landau damping of the beam generated electrostatic waves. Only for values of $\Gamma_{2}<4 \times 10^{-5} \Theta_{-4}$, i.e. very hot pair plasmas and slow Lorentz factors of the outflow, the damping of electrostatic waves outnumbers the initial wave growth.

\subsection{Transverse waves}

Including the contributions from the beam particles to the initial transverse dispersion relation we obtain after analogous manipulations as in the previous subsection

$$
\begin{aligned}
& \Lambda_{\mathrm{t}}(t=0)=1-\frac{c^{2} k^{2}}{\omega^{2}}-\frac{2 \omega_{\mathrm{p}, \mathrm{e}}^{2}}{\omega^{2}-\Omega_{\mathrm{e}}^{2}}-\frac{\omega_{\mathrm{p}, \mathrm{e}}^{2} n_{i}(\omega+k V)}{\omega^{2} \Gamma n_{b}} \\
& {\left[\frac{m_{\mathrm{e}}}{m_{\mathrm{p}}\left(\omega+k V-\frac{m_{\mathrm{e}} \Omega_{\mathrm{e}}}{m_{\mathrm{p}} \Gamma}\right.}+\frac{1}{\omega+k V+\frac{\Omega_{\mathrm{e}}}{\Gamma}}\right]=0}
\end{aligned}
$$

where the two beam contributions stem from the interstellar protons and electrons, respectively. The beam protons will excite transverse waves near their resonance

$\omega_{\mathrm{R}}+k V-\frac{m_{\mathrm{e}} \Omega_{\mathrm{e}}}{m_{\mathrm{p}} \Gamma}=0$

whereas the beam electrons excite transverse waves near

$\omega_{\mathrm{R}}+k V+\frac{\Omega_{\mathrm{e}}}{\Gamma}=0$.

Here we consider only the waves generated by the interstellar protons, and leave the case of electron generated waves to the interested reader. We also assume small wave frequencies, i.e. $\omega^{2} \ll \Omega_{\mathrm{e}}^{2}$ so that Eq. (173) reduces to

$$
\begin{aligned}
& \Lambda_{\mathrm{t}}(t=0) \simeq 1-\frac{c^{2} k^{2}}{\omega^{2}}+\frac{c^{2}}{V_{\mathrm{e}}^{2}} \\
& -\frac{\omega_{\mathrm{p}, \mathrm{e}}^{2} n_{i}}{\omega^{2} \Gamma n_{b}} \frac{\omega+k V}{\omega+k V-\frac{m_{\mathrm{e}} \Omega_{\mathrm{e}}}{m_{\mathrm{p}} \Gamma}}=0 .
\end{aligned}
$$

This approximation holds for wavenumbers $k$ near the proton resonace condition (174). Using $V_{\mathrm{e}}^{2} \ll c^{2}$ we then obtain

$0=\omega^{2}-V_{\mathrm{e}}^{2} k^{2}-\alpha \omega_{\mathrm{p}, \mathrm{e}}^{2} \frac{\omega+k V}{\omega+k V-\frac{m_{\mathrm{e}} \Omega_{\mathrm{e}}}{m_{\mathrm{p}} \Gamma}}$

where we introduce the parameter

$\alpha=\frac{V_{\mathrm{e}}^{2} n_{i}}{c^{2} \Gamma n_{b}}$

Eq. (177) leads to the cubic equation

$\omega^{3}+b \omega^{2}+g \omega+h=0$

with

$b=k V-\Omega, \quad g=-\left(V_{\mathrm{e}}^{2} k^{2}+\alpha \omega_{\mathrm{p}, \mathrm{e}}^{2}\right)$,

$h=k V g+\Omega V_{\mathrm{e}}^{2} k^{2}, \quad \Omega=\frac{m_{\mathrm{e}} \Omega_{\mathrm{e}}}{m_{\mathrm{p}} \Gamma}$.

Provided

$D=Q^{2}+S^{3}>0$,

where

$Q=(b / 3)^{3}-(b g / 6)+(h / 2)=$

$\frac{1}{27}(k V-\Omega)^{3}+\frac{2}{3} k V g-\Omega g+\frac{1}{2} \Omega V_{\mathrm{e}}^{2} k^{2}$

and

$S=(g / 3)-(b / 3)^{2}=-\frac{1}{9}\left[(k V-\Omega)^{2}+3 g\right]$,

the cubic equation (179) has two complex conjugate solution

$\omega=\frac{1}{3}(\Omega-k V)-\frac{1}{2}\left[(-Q+\sqrt{D})^{1 / 3}+(-Q-\sqrt{D})^{1 / 3}\right]$

$\pm \frac{l \sqrt{3}}{2}\left[(-Q+\sqrt{D})^{1 / 3}-(-Q-\sqrt{D})^{1 / 3}\right]$.

The maximum growth rate is a measure for the importance of the instability. For its estimate we use the ansatz of Achatz et al. (1990) that it is largest when the resonance condition (174) is fulfilled yielding with $\omega_{\mathrm{R}}= \pm V_{\mathrm{e}} k$ for relativistic outflow velocities $\left(V \gg V_{\mathrm{e}}\right)$

$k=k_{0}=\frac{\Omega}{V \pm V_{\mathrm{e}}} \simeq \frac{\Omega}{V}$.

For this wavenumber we obtain

$S\left(k_{0}\right)=\frac{1}{3} \alpha \omega_{\mathrm{p}, \mathrm{e}}^{2}[1+3 r], \quad Q\left(k_{0}\right)=\frac{\Omega}{3} \alpha \omega_{\mathrm{p}, \mathrm{e}}^{2}\left[1+\frac{5 r}{2}\right]$ 
with the frequency ratio

$$
\begin{aligned}
& r=\frac{V_{\mathrm{e}}^{2} \Omega^{2}}{\alpha \omega_{\mathrm{p}, \mathrm{e}}^{2} V^{2}}=\frac{2 m_{\mathrm{e}}^{2}}{m_{\mathrm{p}}^{2}} \frac{V_{\mathrm{e}}^{2}}{c^{2} \Gamma\left(\Gamma^{2}-1\right)} \frac{n_{b}^{*}}{n_{i}^{*}} \\
& =5.6 \times 10^{-8} \frac{n_{b, 10}^{*}}{n_{i}^{*}} \epsilon_{\mathrm{B},-1} \Theta_{-4} \Gamma_{2}^{-3}
\end{aligned}
$$

which for standard jet outflow and environment parameters is very small. We then obtain

$\omega_{I}\left(k_{0}\right)=\mathfrak{J} \omega\left(k_{0}\right)=\frac{3^{1 / 2}}{2} D^{1 / 6}\left(k_{0}\right)$

$\times\left[\left(1+\frac{Q\left(k_{0}\right)}{D^{1 / 2}\left(k_{0}\right)}\right)^{1 / 3}+\left(1-\frac{Q\left(k_{0}\right)}{D^{1 / 2}\left(k_{0}\right)}\right)^{1 / 3}\right]$

$\simeq 3^{1 / 2} D^{1 / 6}\left(k_{0}\right) \simeq(\alpha / 3)^{1 / 2}\left[1+\frac{3 \Omega^{2}}{\alpha \omega_{\mathrm{p}, \mathrm{e}}^{2}}\right]^{1 / 6}$

because

$\frac{Q^{2}\left(k_{0}\right)}{D\left(k_{0}\right)}=\frac{r}{r+\left(V_{\mathrm{e}} / V\right)^{2}}<1$

is always smaller than unity.

Collecting terms we obtain

$\omega_{\mathrm{I}}\left(k_{0}\right)=\frac{\omega_{\mathrm{p}, \mathrm{e}}}{\sqrt{3}} \frac{V_{\mathrm{e}}}{c}\left(\frac{n_{i}^{*} \Gamma}{n_{b}^{*}}\right)^{1 / 2}\left[1+0.018 \frac{n_{b, 10}^{*} \Gamma_{2}^{3}}{n_{i}^{*}}\right]$

$=10^{-4} \frac{\Omega_{\mathrm{e}}}{\sqrt{6}}\left(\frac{n_{i}^{*} \Gamma_{2}}{n_{b, 10}^{*}}\right)^{1 / 2}\left[1+0.018 \frac{n_{b, 10}^{*} \Gamma_{2}^{3}}{n_{i}^{*}}\right]$

which has to be compared with the cyclotron damping rate $(154)$ calculated at the wavenumber $k_{0}$ :

$\omega_{\mathrm{I}, \mathrm{A}}\left(k_{0}\right)=-\frac{\pi^{1 / 2}}{2} \Omega_{\mathrm{e}} d_{1} e^{-d_{1}^{2}}$

where $d_{1}=1.3 \times 10^{7} \Gamma_{2} \Theta_{-4}^{-1 / 2}$. Because of the small factor $d_{1} \exp \left(-d_{1}^{2}\right)=\exp \left(-1.7 \times 10^{14}\right)$ the cyclotron damping rate is negligibly small compared to the initial growth rate.

\section{Appendix C: Calculation of the wave spectrum with the bi-spherical shell method}

Here the self-generated wave spectrum of backward moving transverse waves can be evaluated with the assumption, that the free energy (as measured in the plasma rest frame) of the initial beam particle distribution is given to these waves in the isotropization process. This method has been used before for non-relativistic particles when evaluating the wave spectrum generated by freshly ionized pick-up ions (e.g., Huddleston \& Johnstone 1992; Isenberg \& Lee 1996). Generalizing this method to relativistic particles and waves with non-relativistic phase speeds goes straight-forwardly by replacing particle speeds with their momenta.

The final particle distribution is uniform in the so-called (Huddleston \& Johnstone 1992) bi-spherical shell, which in our case of almost Alfvénic waves and particles injected at $p_{\perp}=0$ is accurately given by the spherical shell

$p_{\perp}^{2}+p_{\|}^{2}=p^{2}$,

with the momentum measured in the Alfvén wave frame, where its magnitude, $p$, is taken as constant. (The final distribution function is, thus, isotropic in the Alfvén-wave frame.) We may write the self-generated wave spectrum in form (Isenberg \& Lee 1996)

$I_{\alpha}(k)=4 \pi \frac{\mathrm{d} E}{\mathrm{~d} k} N_{\alpha}\left\{p_{\|}(k)\right\}$,

where $\mathrm{d} E / \mathrm{d} k=\left|\mathrm{d} E / \mathrm{d} p_{\|} \|\right| \mathrm{d} p_{\|} / \mathrm{d} k \mid$ is the energy lost by a particle (per unit resonant wavenumber) as it scatters through the parallel momentum range resonant with wavenumber range $(k, k+\mathrm{d} k)$, and

$N_{\alpha}\left\{p_{\|}(k)\right\}=n_{\alpha} \frac{p-p_{\|}(k)}{2 p}$,

is the number density of particles scattered accross the parallel momentum $p_{\|}(k)$, resonant with $k$, during the isotropization. We have $\left|\mathrm{d} E / \mathrm{d} p_{\|}\right|=V_{\mathrm{e}}$ (with $E$ still measured in the plasma frame, of course), so

$I_{\alpha}(k)=2 \pi n_{\alpha} V_{\mathrm{e}}\left|\frac{\mathrm{d} p_{\|}}{\mathrm{d} k}\right| \frac{p-p_{\|}}{p}$,

where one uses Eq. (86) (and $p_{\|}=p \mu_{i}$ ) evaluated in the Alfvénwave frame to get

$p_{\|}(k) \approx-\frac{m_{\alpha} \Omega_{\alpha}}{k}+\gamma m_{\alpha} V_{\mathrm{e}} \frac{k^{2}}{2 k_{\mathrm{e}}^{2}}$

for the $k$-range of interest to our analysis. Thus, the spectrum reads

$I_{\alpha}(k)=2 \pi n_{\alpha} p V_{\mathrm{e}}\left(\frac{1}{R_{\mathrm{L}, \alpha} k^{2}}+\epsilon_{\alpha} \frac{V_{\mathrm{e}}}{v} \frac{k}{k_{\mathrm{e}}^{2}}\right)\left(1+\frac{\epsilon_{\alpha}}{R_{\mathrm{L}, \alpha} k}\right)$,

where $R_{\mathrm{L}, \alpha}=\gamma v /\left|\Omega_{\alpha}\right|$ is the particle's Larmor radius and $\epsilon_{\alpha}=\operatorname{sign}\left(\Omega_{\alpha}\right)$. The small correction due to the dispersive effects in $\mathrm{d} p_{\|} / \mathrm{d} k$ has to be retained, but the corresponding term in $p-p_{\|}$can be neglected, since it is small in the region $p_{\|} \approx p$.

The above spectrum holds only for a single particle species being picked up by the waves. When several particle species are picked up simultanously, then it may not be possible to avoid considering multiple resonances. For the least massive species the reasoning behind the neglecting of them is still valid, but for the more massive ones, part of the free energy of the lightest species will go to the "denied" bands of the massive particles, which may invalidate the assumption of single resonant wavenumber. In the specific case of electrons and protons, however, the region where multiple resonance are possible for protons, the electron-generated spectrum has a steep slope $\left(\propto k^{-2}\right)$, which as a first approximation still enables us to neglect multiple resonances in case of protons, as well. Thus, the total wave spectrum comes from a contribution from protons and electrons as

$$
\begin{aligned}
I(k)= & 2 \pi \sum_{\alpha} H\left(|k| R_{\mathrm{L}, \alpha}-1\right) n_{\alpha} p_{\alpha} V_{\mathrm{e}}\left(\frac{1}{R_{\mathrm{L}, \alpha} k^{2}}\right. \\
& \left.+\epsilon_{\alpha} \frac{V_{\mathrm{e}}}{v_{\alpha}} \frac{k}{k_{\mathrm{e}}^{2}}\right)\left(1+\frac{\epsilon_{\alpha}}{R_{\mathrm{L}, \alpha} k}\right) .
\end{aligned}
$$




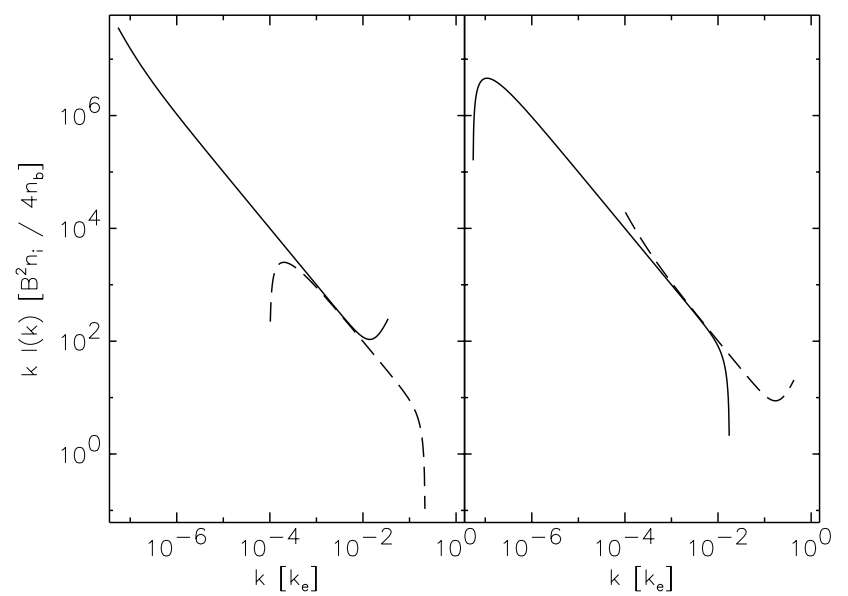

Fig. 2. Spectrum of self-generated waves for after a relativistic pick up of electrons and protons with a $\Gamma=100$. The left [right] panel gives the spectrum of the right-handed [left-handed] waves. Electrongenerated (proton-generated) waves are plotted with a dashed curve (solid curve).

Taking $n_{\alpha}=n_{i}, v_{\alpha}=V, p_{\alpha}=\Gamma m_{\alpha} V$ with $\Gamma=1 / \sqrt{1-(V / c)^{2}}$, and $R_{\mathrm{L}, \alpha}=\Gamma V /\left|\Omega_{\alpha}\right|$, gives

$I(k)=\frac{B^{2}}{4 k_{\mathrm{e}}} \frac{n_{i}}{n_{b}} \sum_{\alpha} H\left(\Gamma V|k|-\Omega_{\alpha}\right)$

$\left(\frac{k_{\mathrm{e}}^{2}}{k^{2}}+\epsilon_{\alpha} \frac{\Gamma m_{\alpha}}{m_{\mathrm{e}}} \frac{k}{k_{\mathrm{e}}}\right)\left(1+\frac{\Omega_{\alpha}}{\Gamma V k}\right)$

showing that the electronic contribution at $\left|\Omega_{\mathrm{e}}\right| / \Gamma V|k| \ll$ $\left(m_{\mathrm{e}} / \Gamma m_{\mathrm{p}}\right)^{1 / 3} k_{\mathrm{e}} \ll k_{\mathrm{e}} / \Gamma^{1 / 3}$ is of the same order as the ionic one. The spectrum and its constituents are plotted in Fig. 2.

\section{Appendix D: Isotropisation by Coulomb interactions}

Apart from scattering with transverse plasma waves additional pitch angle scattering of the plateaued interstellar electrons and protons is provided by the elastic Coulomb interactions with the pair electrons and positrons in the jet outflow plasma. Here we demonstrate that the Coulomb isotropisation time scales of the beam electrons and protons are much longer than the plasma wave isotropisation time scales (106) and (113).

According to Kulsrud et al. (1993) the linearized FokkerPlanck scattering operator for Coulomb interactions is

$\left(\frac{\partial f_{i}}{\partial t}\right)_{\mathrm{C}}=v_{\mathrm{e} i} \frac{\partial}{\partial \mu}\left(1-\mu^{2}\right) \frac{\partial f_{i}}{\partial \mu}$

where the electron scattering rate due to Møller and Bhabha scattering (Böttcher et al. 2001) is

$v_{\mathrm{ee}}=2.6 \times 10^{-3} n_{b, 10}^{*} \Gamma_{2}^{-1} \mathrm{~Hz}$

while the proton scattering rate (Mannheim \& Schlickeiser 1994) is

$v_{\mathrm{ep}}=3.3 \times 10^{-8} n_{b, 10}^{*} \Gamma_{2}^{-1} \mathrm{~Hz}$.
The solution of the associated Fokker-Planck equation during the secular phase

$\left(\frac{\partial f_{i}}{\partial t}\right)=\left(\frac{\partial f_{i}}{\partial t}\right)_{\mathrm{C}}$

with the initial condition (119),

$f_{i}\left(p, \mu, t_{\mathrm{e}}\right)=\frac{1}{2 \pi P p^{2}} \delta(\mu+1) H[P-p]$

is easily obtained in terms of Legrendre polynomials as

$f_{i}\left(p, \mu, t \geq t_{\mathrm{e}}\right)=\frac{H[P-p]}{4 \pi P p^{2}}$

$\times \sum_{n=0}^{\infty}(-1)^{n}(2 n+1) P_{n}(\mu) \exp \left[-n(n+1) v_{\mathrm{e} i}\left(t-t_{\mathrm{e}}\right)\right]$.

For times larger than

$t_{\mathrm{C}, i}=\left(2 v_{\mathrm{e} i}\right)^{-1}$

Eq. (205) approaches the isotropic distribution (120)

$f_{i}(p)=\frac{H[P-p]}{4 \pi P p^{2}}$

here without generating transverse waves.

For electrons the Coulomb isotropisation time scale is

$t_{\mathrm{C}, \mathrm{e}}=1.9 \times 10^{2} \Gamma_{2}\left(n_{b, 10}^{*}\right)^{-1} \mathrm{~s}$

and for protons

$t_{\mathrm{C}, \mathrm{p}}=1.5 \times 10^{7} \Gamma_{2}\left(n_{b, 10}^{*}\right)^{-1} \mathrm{~s}$.

We note that for both particles these time scales are much longer than the time scales (113) and (106) for isotropisation by transverse plasma waves calculated for standard AGN jet outflow and environment parameter

$t_{\mathrm{t}, \mathrm{e}}=3.0 \times 10^{-4} \frac{\left(n_{b, 10}^{*}\right)^{1 / 2}}{n_{i}^{*} \Gamma_{2}^{1 / 6}} \mathrm{~s}$,

and

$t_{\mathrm{t}, \mathrm{p}}=\left(m_{\mathrm{p}} / m_{\mathrm{e}}\right)^{4 / 3} t_{\mathrm{t}, \mathrm{e}}=6.7 \frac{\left(n_{b, 10}^{*}\right)^{1 / 2}}{n_{i}^{*} \Gamma_{2}^{1 / 6}} \mathrm{~s}$.

\section{References}

Achatz, U., Lesch, H., \& Schlickeiser, R. 1990, A\&A, 233, 391 Arbeiter, C., Pohl, M., \& Schlickeiser, R. 2002, A\&A, 386, 415

Atoyan, A., \& Dermer, C. D. 2001, Phys. Rev. Lett., 87, 221102

Bednarek, W., \& Protheroe, R. J. 1999, MNRAS, 302, 373

Bloom, S. D., \& Marscher, A. P. 1996, ApJ, 461, 657

Böttcher, M. 2002, in Gamma-Ray Astrophysics through Multiwavelength Experiments 2001, Bull. Astron. Soc. India, 30, 115

Böttcher, M., Mause, H., \& Schlickeiser, R. 1997, A\&A, 324, 395

Böttcher, M., Schlickeiser, R., \& Marra, A. 2001, ApJ, 563, 71

Buneman, O. 1959, Phys. Rev., 115, 503

Dermer, C. D., \& Schlickeiser, R. 1993, ApJ, 416, 458

Dermer, C. D., Sturner, S. J., \& Schlickeiser, R. 1997, ApJS, 109, 103

Fichtner, H., \& Schlickeiser, R. 1995, Phys. Plasmas, 2, 1063 
Fried, B. D., \& Conte, S. D. 1961, The Plasma Dispersion Function (Academic Press, San Diego)

Hartman, R. C., et al. 1999, ApJS, 123, 79

Huddleston, D. E., \& Johnstone, A. D. 1992, J. Geophys. Res., 97, 12217

Isenberg, P. A., \& Lee, M. A. 1996, J. Geophys. Res., 101, 11055

Kulsrud, R. M., Sun, Y.-C., Winsor, N. K., \& Fallon, H. A. 1973, Phys. Rev. Lett., 31, 690

Lee, M. A., \& Ip, W.-H. 1997, J. Geophys. Res., 92, 11041

Lerche, I., Pohl, M., \& Schlickeiser, R. 2000, J. Plasma Phys., 64, 459

Mannheim, K. 1993, A\&A, 269, 67

Mannheim, K., \& Biermann, P. L. 1992, A\&A, 253, L21

Mannheim, K., \& Schlickeiser, R. 1994, A\&A, 286, 983

Maraschi, L., Ghisellini, G., \& Celotti, A. 1992, ApJ, 397, L5

Pohl, M. 2001, Rapporteur paper at the 27th Intern. Cosmic Ray Conf. [astro-ph/0111552]
Pohl, M., Lerche, I., \& Schlickeiser, R. 2002, A\&A, 383, 309

Pohl, M., \& Schlickeiser, R. 2000, A\&A, 354, 395

Schlickeiser, R. 2002, Cosmic Ray Astrophysics (Springer-Verlag, Heidelberg)

Schlickeiser, R., Fichtner, H., \& Kneller, M. 1997, J. Geophys. Res., 102, 4725

Schlickeiser, R., \& Mause, H. 1995, Phys, Plasmas 2, 4025 102, 4725

Schuster, C., Pohl, M., \& Schlickeiser, R. 2002, A\&A, 382, 829

Sikora, M., Begelman, M. C., \& Rees, M. J. 1994, ApJ, 421, 153

Sikora, M., \& Madejski, G. 2001, in Current High Energy Emission around Black Holes [astro-ph/0112231]

Tavecchio, F., Maraschi, L., \& Ghisellini, G. 1998, ApJ, 509, 608

Vainio, R. 2000, ApJS, 131, 519

Vainio, R., \& Schlickeiser, R. 1999, A\&A, 343, 303

Vainio, R., \& Schlickeiser, R. 2001, A\&A, 378, 309 